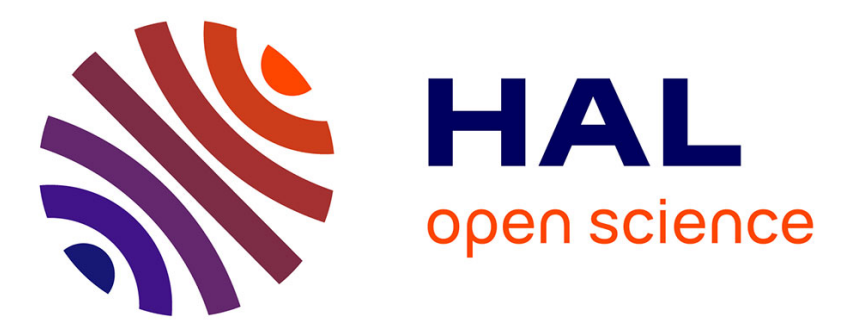

\title{
Zigzag instability of vortex pairs in stratified and rotating fluids. Part 2. Analytical and numerical analyses
}

\author{
P. Billant, A. Deloncle, Jean-Marc Chomaz, P. Otheguy
}

\section{- To cite this version:}

P. Billant, A. Deloncle, Jean-Marc Chomaz, P. Otheguy. Zigzag instability of vortex pairs in stratified and rotating fluids. Part 2. Analytical and numerical analyses. Journal of Fluid Mechanics, 2010, 660 (october), pp.396-429. 10.1017/s002211201000282x . hal-01021123

HAL Id: hal-01021123

https://hal-polytechnique.archives-ouvertes.fr/hal-01021123

Submitted on 16 Jul 2014

HAL is a multi-disciplinary open access archive for the deposit and dissemination of scientific research documents, whether they are published or not. The documents may come from teaching and research institutions in France or abroad, or from public or private research centers.
L'archive ouverte pluridisciplinaire HAL, est destinée au dépôt et à la diffusion de documents scientifiques de niveau recherche, publiés ou non, émanant des établissements d'enseignement et de recherche français ou étrangers, des laboratoires publics ou privés. 


\title{
Zigzag instability of vortex pairs in stratified and rotating fluids. Part 2. Analytical and numerical analyses.
}

\author{
P. BILLANT $\dagger$, A. DELONCLE, J. - M. CHOMAZ \\ AND P. OTHEGUY \\ LadHyX, CNRS, École Polytechnique, F-91128 Palaiseau Cedex, France \\ (Received 10 May 2009; revised 12 May 2010; accepted 13 May 2010; \\ first published online 21 July 2010)
}

The three-dimensional stability of vertical vortex pairs in stratified and rotating fluids is investigated using the analytical approach established in Part 1 and the predictions are compared to the results of previous direct numerical stability analyses for pairs of co-rotating equal-strength Lamb-Oseen vortices and to new numerical analyses for equal-strength counter-rotating vortex pairs. A very good agreement between theoretical and numerical results is generally found, thereby providing a comprehensive description of the zigzag instability. Co-rotating and counter-rotating vortex pairs are most unstable to the zigzag instability when the Froude number $F_{h}=\Gamma /\left(2 \pi R^{2} N\right)$ (where $\Gamma$ is the vortex circulation, $R$ the vortex radius and $N$ the Brunt-Väisälä frequency) is lower than unity independently of the Rossby number $R o=\Gamma /\left(4 \pi R^{2} \Omega_{b}\right)\left(\Omega_{b}\right.$ is the planetary rotation rate). In this range, the maximum growth rate is proportional to the strain $\Gamma /\left(2 \pi b^{2}\right)$ ( $b$ is the separation distance between the vortices) and is almost independent of $F_{h}$ and $R o$. The most amplified wavelength scales like $F_{h} b$ when the Rossby number is large and like $F_{h} b /|R o|$ when $|R o| \ll 1$, in agreement with previous results. While the zigzag instability always bends equal-strength co-rotating vortex pairs in a symmetric way, the instability is only quasi-antisymmetric for finite $R o$ for equal-strength counter-rotating vortex pairs because the cyclonic vortex is less bent than the anticyclonic vortex. The theory is less accurate for co-rotating vortex pairs around $R o \approx-2$ because the bending waves rotate very slowly for long wavelength. The discrepancy can be fully resolved by taking into account higher-order three-dimensional effects.

When $F_{h}$ is increased above unity, the growth rate of the zigzag instability is strongly reduced because the bending waves of each vortex are damped by a critical layer at the radius where the angular velocity of the vortex is equal to the BruntVäisälä frequency. The zigzag instability, however, continues to exist and is dominant up to a critical Froude number, which mostly depends on the Rossby number. Above this threshold, equal-strength co-rotating vortex pairs are stable with respect to longwavelength bending disturbances whereas equal-strength counter-rotating vortex pairs become unstable to a quasi-symmetric instability resembling the Crow instability in homogeneous fluids. However, its growth rate is lower than in homogeneous fluids because of the damping by the critical layer. The structure of the critical layer obtained in the computations is in excellent agreement with the theoretical solution.

Physically, the different stability properties of vortex pairs in stratified and rotating fluids compared to homogeneous fluids are shown to come from the reversal of the direction of the self-induced motion of bent vortices. 
Key words: geophysical and geological flows, instability, vortex flows

\section{Introduction}

Stably stratified flows are encountered in the atmosphere, oceans and lakes. It has been conjectured that these flows should have a two-dimensional, or 'quasi twodimensional', dynamics in the limit of strong stratification since vertical motions are then strongly limited by the buoyancy force (Riley, Metcalfe \& Weissman 1981; Lilly 1983; Lilly et al. 1998; Riley \& Lelong 2000). However, this two-dimensional limit can be reached only if vertical gradients are not too large; otherwise vertical transport terms are not negligible even if vertical velocities are small. The validity of this conjecture has been questioned as many experiments and numerical simulations have shown that strongly stratified turbulent flows are organized into thin horizontal layers with strong vertical variations (see, among others, Herring \& Métais 1989; Park, Whitehead \& Gnanadeskian 1994; Fincham, Maxworthy \& Spedding 1996; Riley \& Lelong 2000; Godeferd \& Staquet 2003; Riley \& deBruynKops 2003; Waite \& Bartello 2004; Praud, Fincham \& Sommeria 2005; Hebert \& deBruynKops 2006; Lindborg 2006; Brethouwer et al. 2007; Lindborg \& Brethouwer 2007). A direct cascade of energy has also been recently reported in numerical simulations of strongly stratified turbulence (Lindborg 2006; Brethouwer et al. 2007) instead of the inverse cascade of energy that should be observed if the dynamics were two-dimensional. Atmospheric and oceanic measurements also support the direct cascade hypothesis (Riley \& Lindborg 2008). On the basis of a self-similarity of the inviscid equations of motion in the limit of strong stratification, Billant \& Chomaz (2001) have shown that the vertical variations should adjust precisely such that vertical and horizontal transport terms are of the same order. In other words, vertical gradients become infinitely large at the same time as the vertical velocity tends to zero when the stratification increases to infinity. The only valid approximation is then the hydrostatic balance along the vertical.

A further reason explaining why stratified flows do not behave like two-dimensional flows is the existence of three-dimensional instabilities (Billant \& Chomaz 2000a,c; Leblanc 2003; Otheguy, Chomaz \& Billant 2006b; Deloncle, Billant \& Chomaz 2008; Waite \& Smolarkiewicz 2008). Because of these instabilities, stratified flows, initially uniform along the vertical, can develop spontaneously strong vertical variations and rapidly evolve towards a three-dimensional state. Leblanc (2003) has shown the existence of a parametric three-dimensional instability of internal waves when they are subjected to an oscillating strain field. Another type of instability is the zigzag instability which bends columnar vertical vortices as a whole with weak core deformations. Such instability has been observed both on equal-strength counterrotating and co-rotating vortex pairs in a strongly stratified fluid (Billant \& Chomaz 2000a,c; Otheguy et al. 2006b; Deloncle et al. 2008; Waite \& Smolarkiewicz 2008). In the case of counter-rotating vortex pairs, the zigzag instability is antisymmetric with respect to the plane separating the vortices. It differs from the Crow instability occurring in homogeneous fluids (Crow 1970), which is characterized by a symmetric bending of the two vortices. It is also different from the elliptic instabilities that distort the inner structure of the vortex cores (Leweke \& Williamson 1998). In the case of co-rotating vortex pairs, the zigzag instability is symmetric (Otheguy et al. 2006b) whereas no such bending instability occurs in homogeneous fluids (Jimenez 
1975; Meunier \& Leweke 2005). The zigzag instability has also been shown to remain active in strongly stratified and rotating fluids whatever the magnitude of the planetary rotation in the cases of an equal strength co-rotating vertical vortex pair (Otheguy, Billant \& Chomaz 2006a) and of an elliptic vortex (Billant, Dritschel \& Chomaz 2006). Thereby, the zigzag instability is of the same nature as the tall-column instability observed by Dritschel \& de la Torre Juárez (1996) in quasi-geostrophic fluids (strongly stratified and rapidly rotating fluids).

The direct numerical stability analyses of an equal-strength co-rotating vortex pair in a stratified fluid performed by Otheguy et al. (2006b) have shown that the zigzag instability is the most dangerous instability when the Froude number is low: $F_{h}=\Gamma /\left(2 \pi R^{2} N\right) \lesssim 1.4-2$, where $R$ is the vortex radius and $N$ the Brunt-Väisälä frequency. (Note that the Froude and Rossby numbers defined by Otheguy et al. $(2006 a, b)$ are twice those used here.) Its growth rate scales like the strain rate $\Gamma /\left(2 \pi b^{2}\right)$, where $\Gamma$ is the vortex circulation and $b$ the separation distance between the vortices, provided that the Froude number $F_{h}$ is less than unity. The corresponding most amplified wavelength scales like $F_{h} b$. These scaling laws have been recovered by Otheguy, Billant \& Chomaz (2007) by means of an asymptotic stability analysis in the case of a strongly stratified fluid $\left(F_{h} \rightarrow 0\right)$. The growth rate of the zigzag instability drops abruptly when $F_{h}>1$ in such a way that it vanishes around $F_{h} \approx 1.4-2$ (Otheguy et al. 2006b). The elliptic instability becomes the most dangerous instability only when the Froude number is much larger: $F_{h} \gtrsim 5$. In the presence of a background rotation, the growth rate of the zigzag instability in the strongly stratified regime, $F_{h}<1$, is almost independent of the Rossby number $R o=\Gamma /\left(4 \pi R^{2} \Omega_{b}\right)$, where $\Omega_{b}$ is the planetary rotation rate (Otheguy et al. 2006a). However, the most amplified wavelength varies with the Rossby number like $F_{h} b / f(R o)$, where the function $f(R o)$ is such that $f(R o) \rightarrow$ constant for $R o \rightarrow \infty$ and $f(R o) \rightarrow R o$ for $R o \rightarrow 0$.

In this paper, we shall explain these scaling laws for any Froude and Rossby numbers using the general theory established in Billant (2010) (hereinafter referred to as Part 1), and it will be shown that similar results apply to equal-strength counter-rotating vortex pairs. New direct numerical stability analysis for the latter configuration have also been carried out in order to validate the theory. The theory describes the coupling between the strain and the bending waves of each columnar vortex for long vertical wavelength and well-separated vortices in a stratified and rotating fluid. It is valid for any Rossby number and when the strain that a vortex exerts on its companion is smaller than the Brunt-Väisälä frequency. The equations turn out to be of the same form as those originally derived by Crow for vortex filaments in homogeneous fluids (Crow 1970). However, the self-induction and mutual-induction functions, which, respectively, describe the effect of the bent vortex on itself and on its companion, are distinctly different in stratified-rotating fluids. Since the theory is valid for any Rossby number and over a wide range of Froude number, we shall be able to provide a comprehensive description of the properties of the zigzag instability and its domain of existence in the $R o-F_{h}$ parameter space. In particular, it will be explained why the zigzag instability for equalstrength co-rotating vortex pairs is suppressed when the Froude number is increased above $F_{h} \approx 1.4-2$ for $R o=\infty$ (Otheguy et al. 2006b). The theory will also provide a general explanation for the physical origin of the zigzag instability and for the differences in the stability of vortex pairs between stratified-rotating and homogeneous fluids.

The outline of the paper is the following. The theory of Part 1 is first briefly summarized in $\S 2.1$ and the numerical method of the direct stability analyses is 
described in $\S 2.2$. In $\S \S 3.1$ and 3.2 , the stability of equal-strength counter-rotating and co-rotating vortex pairs is investigated theoretically and numerically. The stability properties of vortex pairs as a function of the magnitudes of the stratification and planetary rotation are summarized in $\S 3.3$. The stability of unequal-strength vortex pairs is briefly described in $\S 3.4$. In $\S 4$, the central role played by the self-induction function is highlighted. The physical mechanism of the zigzag instability of counterrotating vortex pairs in stratified and rotating fluids compared to the Crow instability in homogeneous fluids is explained heuristically in $\S 4.3$.

\section{Stability problem}

\subsection{Theory}

We consider two columnar vertical vortices of circulation $\Gamma^{(l)}$ and $\Gamma^{(r)}$ and radii $R^{(l)}$ and $R^{(r)}$ separated by a distance $b$ in a rotating stably stratified fluid under the Boussinesq approximation. The Froude number and the Rossby number of each vortex are defined as follows:

$$
F_{h}^{(i)}=\frac{\left|\Gamma^{(i)}\right|}{2 \pi R^{(i)^{2}} N}, \quad R o^{(i)}=\frac{\Gamma^{(i)}}{4 \pi R^{(i)^{2}} \Omega_{b}},
$$

for $i=\{l, r\}$, where $\Omega_{b}$ is the rotation rate about the vertical axis and $N$ the BruntVäisälä frequency. Viscous and diffusive effects are assumed to be small. When the radius of each vortex is small compared to $b$, the two vortices rotate around each other at rate $f=\left(\Gamma^{(l)}+\Gamma^{(r)}\right) /\left(2 \pi b^{2}\right)$, exactly like two point vortices. In the frame of reference rotating at rate $\Omega_{b}+f$, the unperturbed vortex pair is steady. The centre of the vortex $(l)$ lies at $(x, y)=(0,0)$ and the centre of the vortex $(r)$ at $(x, y)=(b, 0)$. If we assume that each vortex is perturbed by long-wavelength bending disturbances (i.e. azimuthal wavenumbers $|m|=1$ ), the positions of the vortex centres in each horizontal plane become

$$
\begin{aligned}
& \left(x^{(l)}, y^{(l)}\right)(z, t)=(0,0)+\operatorname{Re}\left[\left(\Delta x^{(l)}(t), \Delta y^{(l)}(t)\right) \mathrm{e}^{\mathrm{i} \hat{k} z}\right], \\
& \left(x^{(r)}, y^{(r)}\right)(z, t)=(b, 0)+\operatorname{Re}\left[\left(\Delta x^{(r)}(t), \Delta y^{(r)}(t)\right) \mathrm{e}^{\mathrm{i} \hat{k} z}\right],
\end{aligned}
$$

where $\operatorname{Re}$ denotes the real part and $\hat{k}$ the vertical wavenumber. The equations governing the three-dimensional displacement disturbances $\left(\Delta x^{(l)}, \Delta y^{(l)}\right)$ and $\left(\Delta x^{(r)}, \Delta y^{(r)}\right)$ in a stratified-rotating fluid have been derived in Part 1 using the following three assumptions:

$$
R^{(i)} \ll b, \quad \hat{k} F_{h}^{(i)} R^{(i)} \ll \min \left(1,\left|R o^{(i)}\right|\right) \max \left(1, \sqrt{F_{h}^{(i)}}\right), \quad F_{h}^{(i)} \ll\left(\frac{b}{R^{(i)}}\right)^{2},
$$

with $i=\{l, r\}$. The first assumption means that the vortices are well separated. The second hypothesis mostly ensures that the leading three-dimensional effects are always small whatever the Froude and Rossby numbers. In other words, the disturbances have a long vertical wavelength compared to the vortex radius when appropriately scaled by the Froude and Rossby numbers. The last condition is tantamount to specifying that the strains exerted by the vortices on each other are small compared to $N$. It implies that the vortex disturbances are in hydrostatic balance at a distance $b$ from the vortex core. Such a hypothesis is required mainly to obtain analytically the effect of the disturbances of one vortex on its companion in stratified-rotating 
fluids. Under the above hypotheses, the displacement perturbations of the vortex with superscript $(l)$ are governed by

$$
\begin{aligned}
& \frac{\partial \Delta x^{(l)}}{\partial t}=-\frac{\Gamma^{(r)}}{2 \pi b^{2}} \Delta y^{(l)}+\frac{\Gamma^{(r)}}{2 \pi b^{2}} \Psi \Delta y^{(r)}+\left(f-\frac{\Gamma^{(l)}}{2 \pi R^{(l)^{2}}} \omega_{r}^{(l)}\right) \Delta y^{(l)}+\frac{\left|\Gamma^{(l)}\right|}{2 \pi R^{(l)^{2}}} \omega_{i}^{(l)} \Delta x^{(l)}, \\
& \frac{\partial \Delta y^{(l)}}{\partial t}=-\frac{\Gamma^{(r)}}{2 \pi b^{2}} \Delta x^{(l)}+\frac{\Gamma^{(r)}}{2 \pi b^{2}} \chi \Delta x^{(r)}-\left(f-\frac{\Gamma^{(l)}}{2 \pi R^{(l)^{2}}} \omega_{r}^{(l)}\right) \Delta x^{(l)}+\frac{\left|\Gamma^{(l)}\right|}{2 \pi R^{(l)^{2}}} \omega_{i}^{(l)} \Delta y^{(l)} .
\end{aligned}
$$

The corresponding equations for the displacement perturbations of the vortex $(r)$ have the same form with the superscripts $(r)$ and $(l)$ interchanged. These equations are formally the same as in homogeneous fluids (Crow 1970; Jimenez 1975; Bristol et al. 2004). As explained in Part 1, the first term on the right-hand sides of (2.5)-(2.6) is the strain effect due to the companion vortex. The second term represents mutualinduction effects, i.e. the effect of the perturbation of the companion vortex. This effect depends on the first and second mutual-induction functions $\chi$ and $\Psi$. Under the hypotheses (2.4), these functions are the following:

$$
\begin{aligned}
\chi & =\hat{\beta} b K_{1}(\hat{\beta} b)+\hat{\beta}^{2} b^{2} K_{0}(\hat{\beta} b), \\
\Psi & =\hat{\beta} b K_{1}(\hat{\beta} b),
\end{aligned}
$$

where $\hat{\beta}=2 \hat{k}\left|\Omega_{b}\right| / N$. The third term on the right-hand side of (2.5)-(2.6) comes from the orbital rotation of the vortex pair. Finally, the fourth and fifth terms correspond to the effect of the vortex $(l)$ on itself as if it were alone. They depend on the self-induction function, which, for a stratified-rotating fluid, is given by

$$
\omega^{(i)}=\omega_{r}^{(i)}+\mathrm{i} \omega_{i}^{(i)}=\frac{\hat{\beta}^{2} R^{(i)^{2}}}{2}\left[-\ln \left(\frac{\hat{\beta} R^{(i)}}{2}\right)+\delta\left(F_{h}^{(i)}, R o^{(i)}\right)-\gamma_{e}\right],
$$

where $\gamma_{e}=0.5772 \ldots$ is Euler's constant and

$$
\delta\left(F_{h}, R o\right)=\mathscr{D}\left(F_{h}\right)+2 R o \mathscr{B}\left(F_{h}\right)+R o^{2} \mathscr{A}\left(F_{h}\right) .
$$

The parameters $(\mathscr{A}, \mathscr{B}, \mathscr{D})$ are constants depending on $F_{h}$ and the vortex profile

$$
\begin{aligned}
& \mathscr{A}\left(F_{h}\right)=\lim _{\eta_{0} \rightarrow \infty} \int_{0}^{\eta_{0}} \frac{\xi^{3} \Omega(\xi)^{4}}{1-F_{h}^{2} \Omega(\xi)^{2}} \mathrm{~d} \xi-\frac{1}{4 F_{h}^{2}} \ln \left(1-\frac{F_{h}^{2}}{\eta_{0}^{4}}\right), \\
& \mathscr{B}\left(F_{h}\right)=\lim _{\eta_{0} \rightarrow \infty} \int_{0}^{\eta_{0}} \frac{\xi^{3} \Omega(\xi)^{3}}{1-F_{h}^{2} \Omega(\xi)^{2}} \mathrm{~d} \xi-\frac{1}{4 F_{h}} \ln \left(\frac{\eta_{0}^{2}-F_{h}}{\eta_{0}^{2}+F_{h}}\right), \\
& \mathscr{D}\left(F_{h}\right)=\lim _{\eta_{0} \rightarrow \infty} \int_{0}^{\eta_{0}} \frac{\xi^{3} \Omega(\xi)^{2}}{1-F_{h}^{2} \Omega(\xi)^{2}} \mathrm{~d} \xi-\frac{1}{4} \ln \left(\eta_{0}^{4}-F_{h}^{2}\right),
\end{aligned}
$$

where $\Omega(r)$ is the distribution of angular velocity of each vortex (i) nondimensionalized by $\Gamma^{(i)} /\left(2 \pi R^{(i)^{2}}\right)$ and $r$ is the radius non-dimensionalized by the vortex radius $R^{(i)}$. When $F_{h}>1 / \Omega_{\max }$, where $\Omega_{\max }$ is the maximum angular velocity of the vortex, the denominators in the above integrals exhibit a singularity at the radius $r_{c}$, where $\Omega\left(r_{c}\right)=1 / F_{h}$. If viscous and strain effects smooth this critical layer, the singularity has to be avoided by deforming the contour of integration in the upper complex plane (see Part 1). Accordingly, the coefficients $(\mathscr{A}, \mathscr{B}, \mathscr{D})$ become complex with a negative imaginary part. 
In this paper, we consider vortex pairs whose individual vortices have a LambOseen profile:

$$
\Omega=\frac{1}{r^{2}}\left(1-\mathrm{e}^{-r^{2}}\right) .
$$

The parameters $\mathscr{A}, \mathscr{B}$ and $\mathscr{D}$ for this profile are displayed in figure 4 of Part 1 as a function of the Froude number. As shown in Part 1, the self-induction function $\omega^{(i)}$ for (2.14) is real and positive whatever the Rossby number for $F_{h}^{(i)}<1 / \Omega_{\max }=1$. When $F_{h}^{(i)}>1, \omega^{(i)}$ becomes complex with a negative imaginary part.

Now returning to the description of the self-induction effect in (2.5)-(2.6), the fourth term on the right-hand side involves the real part of the self-induction function $\omega_{r}^{(l)}$ and corresponds to a rigid rotation of the sinusoidally bent vortex around its unperturbed position. The fifth term depends on the imaginary part of the self-induction function $\omega_{i}^{(l)}$ and represents a damping of the bending disturbance when there is a critical layer.

The leading viscous and diffusive effects can also be easily taken into account when there is no critical layer. The self-induction function (2.9) is then modified as follows:

$$
\omega^{(i)} \rightarrow \omega^{(i)}-\mathrm{i} \frac{\hat{k}^{2} R^{(i)^{2}}}{R e^{(i)}} \mathscr{V}\left(F_{h}^{(i)}, R o^{(i)}, S c\right),
$$

where $R e^{(i)}=\left|\Gamma^{(i)}\right| /(2 \pi \nu)$ are the Reynolds numbers, $S_{c}=v / D$ is the Schmidt number and $\mathscr{V}$ is a constant defined in Part 1 , which depends on the vortex profile, and the Froude, Rossby and Schmidt numbers.

In order to investigate the stability of vortex pairs, the displacement perturbations are written in the form

$$
\left(\Delta x^{(l)}, \Delta y^{(l)}, \Delta x^{(r)}, \Delta y^{(r)}\right)=\bar{\Delta} x \mathrm{e}^{\hat{\sigma} t},
$$

where $\hat{\sigma}$ is the growth rate and $\bar{\Delta} \boldsymbol{x} \equiv\left(\bar{\Delta} x^{(l)}, \bar{\Delta} y^{(l)}, \bar{\Delta} x^{(r)}, \bar{\Delta} y^{(r)}\right)$ the eigenvector. Then, (2.5)-(2.6) and the complementary equations for the vortex $(r)$ give the following dispersion relation:

$$
\begin{aligned}
& \left(\sigma-\tilde{b}^{2} \omega_{i}^{(l)}\right)^{2}\left(\sigma-|\tilde{\Gamma}| \tilde{b}^{2} \tilde{R}^{2} \omega_{i}^{(r)}\right)^{2}+\left[\left(\tilde{\Gamma} \tilde{f}-\tilde{\Gamma} \tilde{b}^{2} \tilde{R}^{2} \omega_{r}^{(r)}\right)^{2}-1\right]\left(\sigma-\tilde{b}^{2} \omega_{i}^{(l)}\right)^{2} \\
& \quad+\left[\left(\tilde{\Gamma} \tilde{f}-\tilde{b}^{2} \omega_{r}^{(l)}\right)^{2}-\tilde{\Gamma}^{2}\right]\left(\sigma-|\tilde{\Gamma}| \tilde{b}^{2} \tilde{R}^{2} \omega_{i}^{(r)}\right)^{2}-2 \chi \Psi \tilde{\Gamma}\left(\sigma-\tilde{b}^{2} \omega_{i}^{(l)}\right) \\
& \quad \times\left(\sigma-|\tilde{\Gamma}| \tilde{b}^{2} \tilde{R}^{2} \omega_{i}^{(r)}\right)+\left[\tilde{\Gamma} \Psi^{2}-\left(\tilde{\Gamma} \tilde{f}-\tilde{\Gamma} \tilde{b}^{2} \tilde{R}^{2} \omega_{r}^{(r)}-1\right)\left(\tilde{\Gamma} \tilde{f}-\tilde{b}^{2} \omega_{r}^{(l)}-\tilde{\Gamma}\right)\right] \\
& \quad \times\left[\tilde{\Gamma} \chi^{2}-\left(\tilde{\Gamma} \tilde{f}-\tilde{\Gamma} \tilde{b}^{2} \tilde{R}^{2} \omega_{r}^{(r)}+1\right)\left(\tilde{\Gamma} \tilde{f}-\tilde{b}^{2} \omega_{r}^{(l)}+\tilde{\Gamma}\right)\right]=0,
\end{aligned}
$$

where $\sigma=\hat{\sigma} 2 \pi b^{2} / \Gamma^{(l)}$ is the non-dimensional growth rate scaled by the strain of the left vortex, $\tilde{\Gamma}=\Gamma^{(r)} / \Gamma^{(l)}$ is the circulation ratio, $\tilde{f}=1+1 / \tilde{\Gamma}, \tilde{R}=R^{(l)} / R^{(r)}$ and $\tilde{b}=b / R^{(l)}$ is the separation ratio. In the present paper, we shall restrict the stability analysis mainly to the cases of equal-strength co-rotating and counter-rotating vortex pairs $\tilde{\Gamma}= \pm 1$ with the same radius $R^{(l)}=R^{(r)} \equiv R$. The circulation of the vortex $(l)$ is taken positive by convention.

\subsection{Direct numerical stability analyses}

The theoretical results will be compared to the results of direct numerical stability analyses of pairs of adapted Lamb-Oseen vortices. For equal-strength co-rotating vortex pairs, we shall use the results already obtained by Otheguy et al. $(2006 a, b)$ for the separation ratio $\tilde{b}=6.7$. Additional stability analyses have also been carried 
out for $\tilde{b}=15$. For counter-rotating vortex pairs, new stability analyses have been performed for the separation ratio $\tilde{b}=8$ using the same numerical method as in Otheguy et al. (2006b). In this method, the basic state is first computed by integrating the two-dimensional Navier-Stokes equations initialized by two Lamb-Oseen vortices using a pseudospectral method with periodic boundary conditions. Time advancement is carried out with the fourth-order Runge-Kutta scheme for the nonlinear term and exact integration for the viscous and diffusive terms. Most of the aliasing errors are eliminated by truncating $95 \%$ of the modes along each direction. After a short integration time, the vortices adapt to each other and evolve towards a quasisteady state. The three-dimensional Navier-Stokes equations under the Boussinesq approximation linearized around this basic state are then integrated for each vertical wavenumber with a white noise as initial conditions and using the same pseudospectral code. After a sufficiently long integration time, the perturbation consists solely of the eigenmode with the largest growth rate. More details on the numerical method can be found in Otheguy et al. (2006b) and Billant \& Chomaz (2000c).

The resolution used in this paper is $640 \times 512$ in the horizontal plane. The horizontal size of the computational domain is chosen sufficiently large: $L_{x}=38 R, L_{y}=30 R$ for $\tilde{b}=8$ and $L_{x}=75 R, L_{y}=60 R$ for $\tilde{b}=15$, in order to minimize the effects of the periodic boundary conditions. Their effects are tested in the Appendix and are shown to be very small. The time step is $\delta t=0.012 \pi R^{2} / \Gamma$. Several tests using different resolutions, time steps and box sizes have been carried out in order to check the accuracy of the computations. The Schmidt number $S c$ is set to unity: $S_{c}=v / D=1$. For most computations, the Reynolds number is $R e=50000$, i.e. large enough to be close to the inviscid limit of the theory. Smaller and larger values of $R e$ will also be investigated in $\S \S 3.1 .2$ and 3.1.3. We refer to Otheguy et al. $(2006 a, b)$ for the numerical parameters used for the case of co-rotating vortex pairs with $\tilde{b}=6.7$.

\section{Results}

\subsection{Stability of an equal-strength counter-rotating vortex pair}

When the two vortices have opposite circulation, $\tilde{\Gamma}=-1$, their Froude numbers are equal, i.e. $F_{h}^{(l)}=F_{h}^{(r)} \equiv F_{h}$, but their Rossby numbers are opposite because one vortex is a cyclone $\left(R o^{(l)} \equiv R o>0\right)$ and the other an anticyclone $\left(R o^{(r)}=-R o<0\right)$. Their self-induction functions are then not equal since they depend on the sign of the Rossby number. This implies that symmetric eigenvector defined by

$$
\overline{\boldsymbol{\Delta}} \boldsymbol{x}_{s}=\frac{1}{2}\left[\bar{\Delta} x_{s}, \bar{\Delta} y_{s},-\bar{\Delta} x_{s}, \bar{\Delta} y_{s}\right],
$$

with $\bar{\Delta} x_{s}=\bar{\Delta} x^{(l)}-\bar{\Delta} x^{(r)}, \bar{\Delta} y_{s}=\bar{\Delta} y^{(l)}+\bar{\Delta} y^{(r)}$, and antisymmetric eigenvector

$$
\overline{\boldsymbol{\Delta}} \boldsymbol{x}_{a}=\frac{1}{2}\left[\bar{\Delta} x_{a}, \bar{\Delta} y_{a}, \bar{\Delta} x_{a},-\bar{\Delta} y_{a}\right],
$$

with $\bar{\Delta} x_{a}=\bar{\Delta} x^{(l)}+\bar{\Delta} x^{(r)}, \bar{\Delta} y_{a}=\bar{\Delta} y^{(l)}-\bar{\Delta} y^{(r)}$, are not decoupled for finite $R o$ in contrast to homogeneous fluids (Crow 1970). In the latter case, the normal modes separate into two classes because equal-strength counter-rotating vortex pairs have a mirror symmetry with respect to the plane midway between the two vortex centres. The symmetric mode is the mode which preserves this symmetry while the antisymmetric mode has the opposite symmetry. However, this symmetry of the base state no longer exists when there is a background rotation. A decoupling between symmetric and antisymmetric modes is recovered only for $R o=\infty$ or when the background rotation is rapid $(R o \rightarrow 0)$ because the self-induction functions of the two vortices are equal 


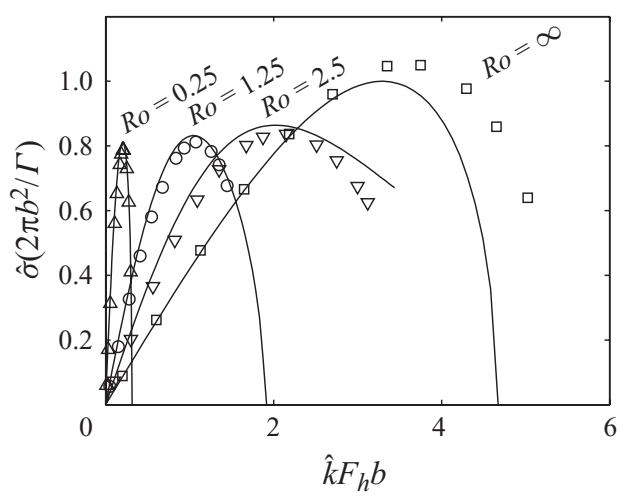

FiguRE 1. Growth rate of the zigzag instability as a function of the rescaled vertical wavenumber $\hat{k} F_{h} b$ for two equal-strength counter-rotating Lamb-Oseen vortices with $\tilde{b}=8$ for $F_{h}=0.1$, and for various Rossby numbers. The solid lines show the theoretical growth rate (cf. (2.17)) for $R e=\infty$ and the symbols show the results of a direct numerical stability analysis for $R e=50000$, and for $R o=\infty(\square), R o=2.5(\nabla), R o=1.25(\bigcirc)$ and $R o=0.25(\triangle)$.

in these limits: $\omega^{(l)}=\omega^{(r)} \equiv \omega$. The dispersion relation (2.17) then simplifies and yields the formulae of Crow (1970) for the growth rates of symmetric and antisymmetric modes:

$$
\begin{aligned}
& \left(\sigma_{s}-\tilde{b}^{2} \omega_{i}\right)^{2}=\left(1+\tilde{b}^{2} \omega_{r}+\chi\right)\left(1-\tilde{b}^{2} \omega_{r}-\Psi\right), \\
& \left(\sigma_{a}-\tilde{b}^{2} \omega_{i}\right)^{2}=\left(1+\tilde{b}^{2} \omega_{r}-\chi\right)\left(1-\tilde{b}^{2} \omega_{r}+\Psi\right),
\end{aligned}
$$

which are denoted by $\sigma_{s}$ and $\sigma_{a}$, respectively.

\subsubsection{Effect of the Rossby number for a strongly stratified fluid}

Figure 1 shows the theoretical growth rate in the inviscid limit (solid lines) as a function of the rescaled vertical wavenumber $\hat{k} F_{h} b$ in a stratified-rotating fluid for $\tilde{b}=8, F_{h}=0.1$ and for several Rossby numbers. Only positive Rossby numbers are displayed because the growth rate is independent of the sign of $R o$ since the two vortices are of opposite sign. We see that the growth rate is maximum for a finite vertical wavenumber. The maximum growth rate is around unity for all the Rossby numbers but the most amplified rescaled vertical wavenumber $\hat{k} F_{h} b$ is shifted towards small values when $R o$ decreases. The theoretical results are in excellent agreement with the numerical results (symbols) for all the Rossby numbers. The discrepancies observed at large wavenumbers for $R o=\infty$ and $R o=2.5$ are due to the long-wavelength assumption used in the theory. The slight departures seen at small wavenumbers for $R o=2.5$ and $R o=1.25$ are shown in the Appendix to come from the periodic boundary conditions used in the numerical stability analysis.

Figure $2(b)$ shows the vertical vorticity of the theoretically most amplified eigenmode for $R o=\infty$. It is given at leading order by (Part 1)

$$
\tilde{\zeta}=-\bar{\Delta} x^{(l)} \frac{\partial \hat{\zeta}^{(l)}}{\partial x}-\bar{\Delta} y^{(l)} \frac{\partial \hat{\zeta}^{(l)}}{\partial y}-\bar{\Delta} x^{(r)} \frac{\partial \hat{\zeta}^{(r)}}{\partial x}-\bar{\Delta} y^{(r)} \frac{\partial \hat{\zeta}^{(r)}}{\partial y},
$$

where $\hat{\zeta}^{(i)}$ is the dimensional basic vertical vorticity of each vortex. As indicated by the arrows in figure $2(b)$, this mode induces antisymmetric displacements of the vortices: $\left(\bar{\Delta} x^{(l)}, \bar{\Delta} y^{(l)}\right)=\left(\bar{\Delta} x^{(r)},-\bar{\Delta} y^{(r}\right)=(0.498,0.502)$. This mode is similar to the eigenmode of the zigzag instability for the Lamb-Chaplygin counter-rotating vortex pair (Billant \& 
(a)

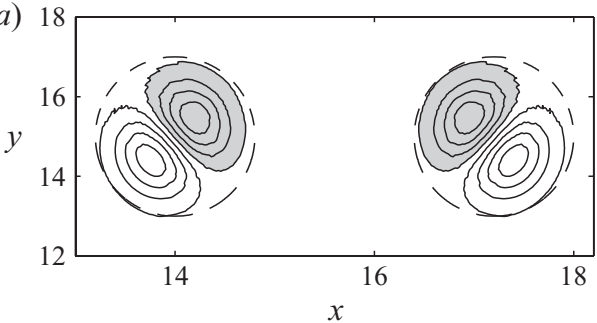

(b)

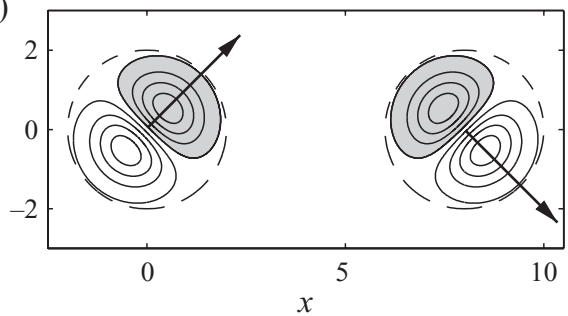

Figure 2. Comparison between the numerical $(a)$ and theoretical $(b)$ vertical vorticities of the most amplified eigenmode of two equal-strength counter-rotating vortices for $R o=\infty, F_{h}=0.1$ and $\tilde{b}=8$. The theoretical and numerical eigenmodes have been obtained for $R e=\infty$ and $R e=50000$, respectively. Shaded areas are regions of positive values. In each plot, the contour level is $24 \%$ of the maximum value. The dotted circles show the location of the basic vortices and have a radius $2 R$. The arrows in $(b)$ indicate the directions of displacement of each vortex. The labels of the $x$ and $y$ axes are expressed in radius unit $R$. In $(a)$, only a domain of size $13 R \times 6 R$ is shown while the computational domain is $38 R \times 30 R$. The $x$ and $y$ labels are different in $(a)$ and $(b)$ because the origin is set at the bottom left corner of the computational domain in $(a)$ whereas it is set at the centre of the left vortex in $(b)$.
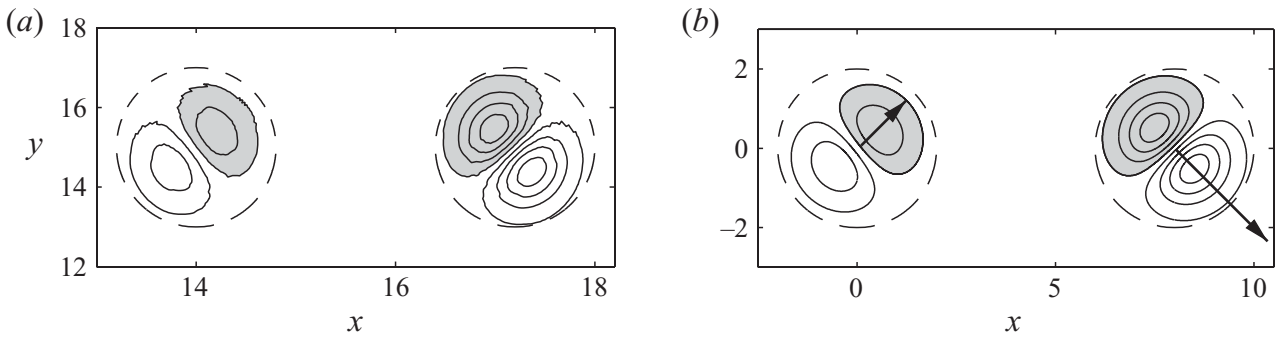

FiguRE 3. Similar to figure 2 but for $R o=1.25$.

Chomaz 2000c). As shown in figure 2(a), the theoretical eigenmode fully agrees with the eigenmode obtained in the numerical stability analysis. The symmetric eigenmode (3.1) is stable for $F_{h}=0.1$ and $R o=\infty$ in contrast to homogeneous fluids where it is unstable and known as the Crow instability (Crow 1970).

For finite $R o$, the most amplified eigenmode is similar except that there is now an asymmetry between the two vortices (figure 3): the left vortex which is cyclonic is less displaced than the right vortex which is an anticyclone. (For the value $R o=1.25$ shown in figure 3 , these displacements are $\left(\bar{\Delta} x^{(l)}, \bar{\Delta} y^{(l)}\right)=(0.35,0.27)$ and $\left(\bar{\Delta} x^{(r)}, \bar{\Delta} y^{(r}\right)=(0.61,-0.66)$.) This difference is due to the fact that the selfinduction of the cyclone is higher than that of the anticyclone for a given vertical wavenumber. Nevertheless, this eigenmode can be called 'quasi-antisymmetric' since the displacements remain mostly antisymmetric and furthermore becomes purely antisymmetric in the long-wavelength limit $\hat{k} \rightarrow 0$. The agreement between the numerically computed eigenmode (figure $3 a$ ) and the theoretical eigenmode (figure $3 b$ ) is excellent. Figure 4(a) further shows the antisymmetric and symmetric components of the displacements of the theoretical and most amplified eigenmode as a function of $R o$ for $F_{h}=0.1$. We see that the symmetric components (dotted and dasheddotted lines) tend to zero in the limits $R o \rightarrow 0$ and $R o \rightarrow \infty$ and are maximum for $R o \approx 2$. However, they always remain smaller than the antisymmetric components (solid and dashed lines). It is also worth noting that the antisymmetric displacements 

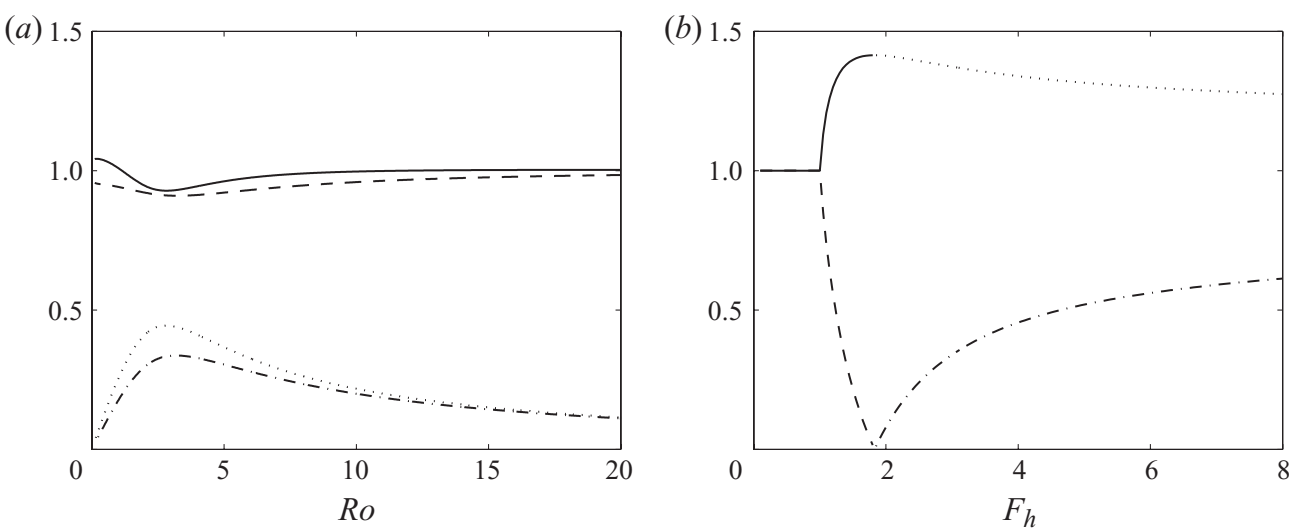

FiguRE 4. Displacements of the theoretical most amplified disturbance of two equal-strength counter-rotating Lamb-Oseen vortices with $\tilde{b}=8$ and $R e=\infty(a)$ as a function of the Rossby number $R o$ for $F_{h}=0.1$ and $(b)$ as a function of the Froude number $F_{h}$ for $R o=\infty$. The different lines correspond to the $x$ and $y$ components of the antisymmetric mode: $\bar{\Delta} x_{a}(-)$, $\bar{\Delta} y_{a}(---)$ and of the symmetric mode: $\bar{\Delta} x_{s}(-\cdot-), \bar{\Delta} y_{s}(\cdots)$.
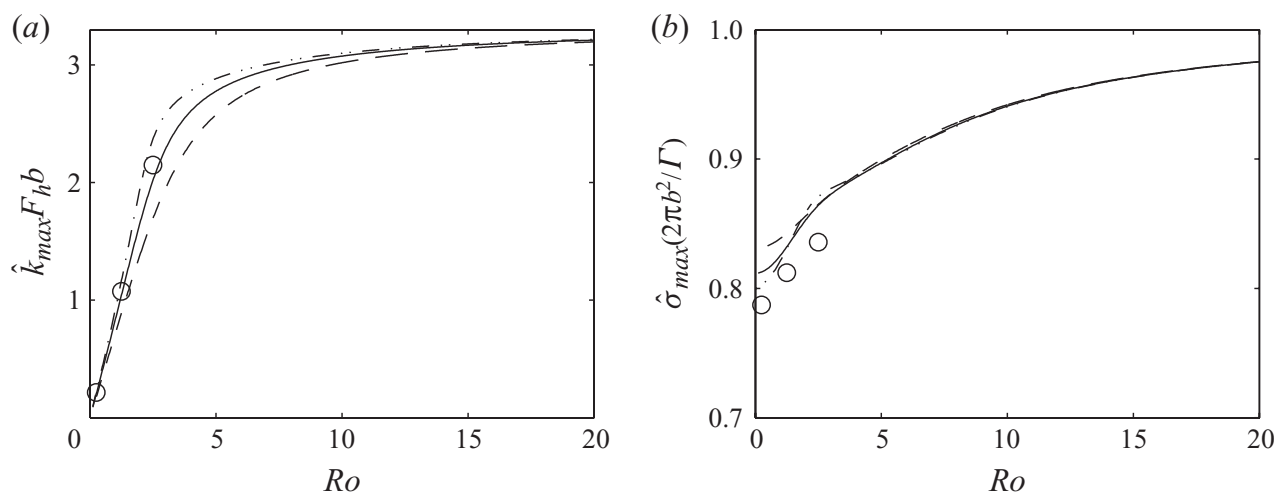

FiguRE 5. Most amplified vertical wavenumber $(a)$ and the maximum growth rate $(b)$ of the zigzag instability of two equal-strength counter-rotating Lamb-Oseen vortices for $F_{h}=0.1$ as a function of the Rossby number. The curves show the inviscid theoretical predictions for $\tilde{b}=6(-\cdot-), \tilde{b}=8(-)$ and $\tilde{b}=16(---)$. The symbols $(\bigcirc)$ show the results of a direct-numerical-stability analysis for $F_{h}=0.1, \tilde{b}=8$ and $R e=50000$.

in the $x$ and $y$ directions are approximately equal. The same feature is observed for the symmetric displacements. This means that each vortex is displaced in a direction making approximately a $45^{\circ}$ angle with the $x$-axis whatever be $R o$. Only the amplitudes of the displacements vary with $R o$, and differ between the cyclonic and anticyclonic vortices, as observed in figure 3.

Figure 5(a) shows that the most amplified wavenumber scaled by $b F_{h}$ decreases monotonically with decreasing $R o$ for a fixed Froude number $F_{h}=0.1$. For large $R o, \hat{k}_{\text {max }} F_{h} b$ is constant and for small $R o$, it scales like $R o$, in agreement with the quasi-geostrophic theory. The maximum growth rate is approximately equal to unity (i.e. the dimensional growth rate is approximately equal to the strain) independently of $R o$ (figure $5 b$ ). Figure 5 also demonstrates that the maximum growth rate and the corresponding rescaled wavenumber remain almost constant if the separation ratio $\tilde{b}$ is varied from 6 to 16 . 

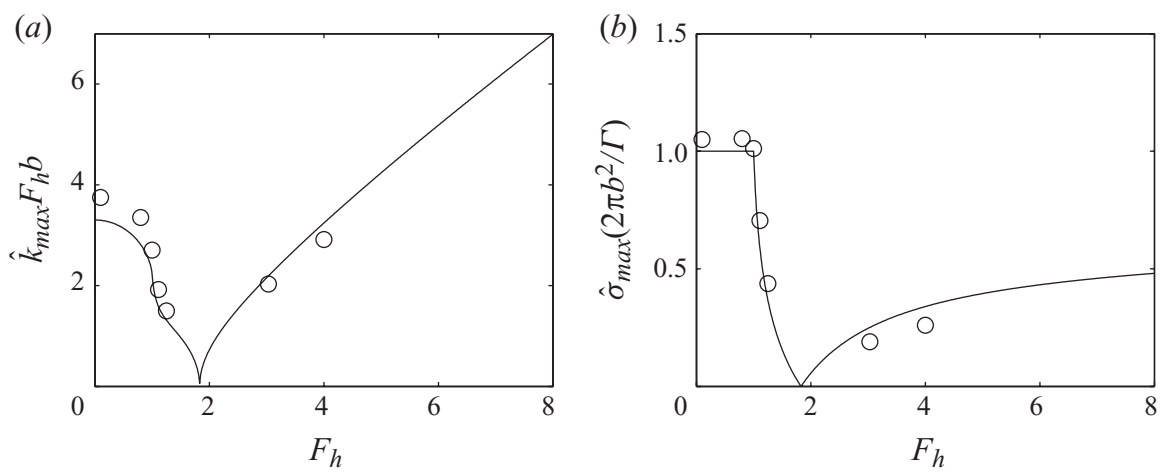

FIGURE 6. Most amplified vertical wavenumber $(a)$ and the maximum growth rate $(b)$ of two equal-strength counter-rotating Lamb-Oseen vortices with $\tilde{b}=8$ as a function of the Froude number for $R o=\infty$. The solid lines show the inviscid theoretical prediction and the symbols show the results of a direct numerical stability analysis for $R e=50000$.

These scaling laws can be easily understood by noticing that the mutual-induction functions depend only on $\hat{\beta} b=\hat{k} F_{h} b /|R o|$ whereas the self-induction terms in (2.17), i.e. $\tilde{b}^{2} \omega^{(i)}$, can be expressed in terms of four independent variables: $\hat{\beta} b$, the separation ratio $\tilde{b}, R o$ and $F_{h}$. However, the separate dependence on $\tilde{b}$ is only logarithmic and the dependences on $R o$ and $F_{h}$ are weak for small $R o$ and $F_{h}<1$. This is the reason why the effect of varying $\tilde{b}$ is weak (figure 5) and why the growth rate depends mostly on $\hat{k} F_{h} b /|R o|$ for small $R o$ and $F_{h}$. In the limit $R o \rightarrow \infty, \tilde{b}^{2} \omega^{(i)}$ depends only on $\hat{k} F_{h} b$ for small $F_{h}$ so that varying the separation ratio $\tilde{b}$ or $F_{h}$ has no effect on the maximum growth rate and the most amplified rescaled wavenumber $\hat{k} F_{h} b$.

\subsubsection{Effect of the Froude number for a non-rotating fluid}

We now investigate the effect of the Froude number for a non-rotating fluid: $R o=\infty$. In this case, symmetric and antisymmetric modes are decoupled and their growth rates (see (3.3)-(3.4)) become particularly simple since $\Psi=\chi=1$ for all $\hat{k}$ :

$$
\begin{aligned}
& \left(\sigma_{s}-\tilde{b}^{2} \omega_{i}\right)^{2}=-\left(2+\tilde{b}^{2} \omega_{r}\right) \tilde{b}^{2} \omega_{r}, \\
& \left(\sigma_{a}-\tilde{b}^{2} \omega_{i}\right)^{2}=\left(2-\tilde{b}^{2} \omega_{r}\right) \tilde{b}^{2} \omega_{r},
\end{aligned}
$$

and the self-induction function is $\omega=\hat{k}^{2} R^{2} F_{h}^{2} \mathscr{A}\left(F_{h}\right) / 2$. The maximum growth rate and the most amplified wavenumber of the unstable mode, either symmetric or antisymmetric, can be derived easily:

$$
\sigma_{\text {max }}=\frac{|\mathscr{A}|+\mathscr{A}_{i}}{\left|\mathscr{A}_{r}\right|}, \quad \hat{k}_{\text {max }} F_{h} b=\sqrt{\frac{2}{\left|\mathscr{A}_{r}\right|}} \sqrt{1-\frac{\left|\mathscr{A}_{i}\right|}{|\mathscr{A}|}},
$$

where $\mathscr{A}_{r}=\operatorname{Re}(\mathscr{A})$ and $\mathscr{A}_{i}=\operatorname{Im}(\mathscr{A})$. As can be seen in figure $6(b)$, the theoretical maximum growth rate (solid line) remains constant and equal to unity when $F_{h} \leqslant 1$, but when $F_{h}$ is increased above unity, it drops abruptly and goes to zero for $F_{h}=1.83$. It rises again slowly when $F_{h}$ is increased further. The corresponding most amplified scaled wavenumber $\hat{k}_{\max } F_{h} b$ also decreases almost to zero for $F_{h}=1.83$ and then increases linearly with $F_{h}$ (figure $6 a$ ). The agreement between the theory and the numerical results (symbols) is very good for all the investigated values of $F_{h}$.

These behaviours can be simply understood from (3.6)-(3.8). For $F_{h} \leqslant 1$, the selfinduction $\omega$ is purely real and positive, i.e. $\omega_{r}>0$ and $\omega_{i}=0$ (or equivalently $\mathscr{A}_{r}>0$ and $\mathscr{A}_{i}=0$ ). Hence, the symmetric mode (3.6) is stable while the antisymmetric 
(a)

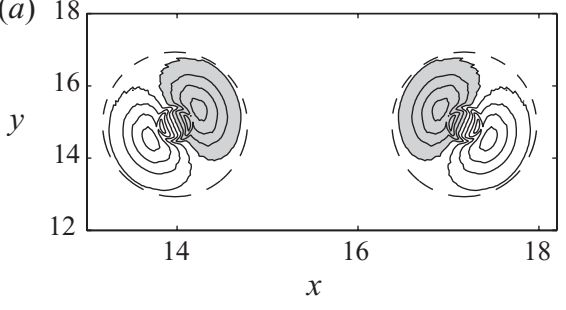

(b)

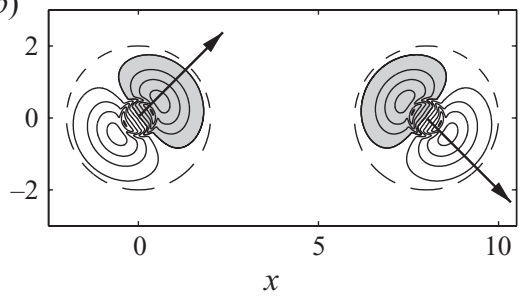

Figure 7. Similar to figure 2 but for $F_{h}=1.11$.
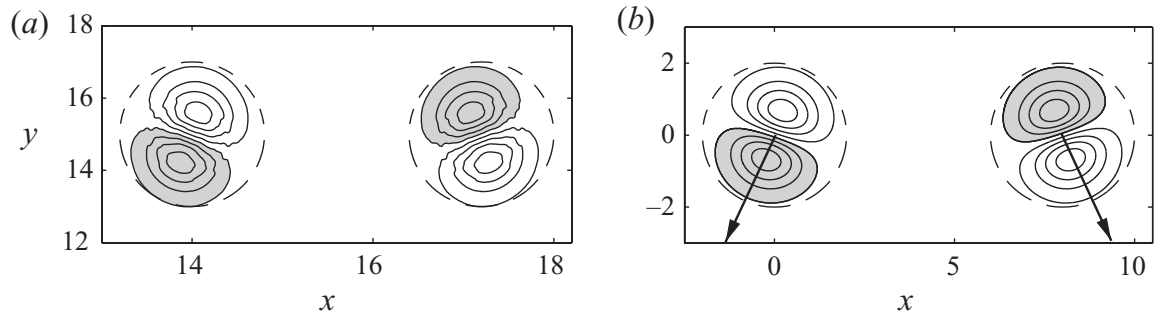

FiguRE 8. Similar to figure 2 but for $F_{h}=4$.

mode (3.7) is unstable for $0<\tilde{b}^{2} \omega_{r}<2$. Its maximum growth rate $\sigma_{\max }=1$ is reached when $\tilde{b}^{2} \omega_{r}=1$. When $F_{h}>1$, the self-induction $\omega$ becomes complex with a negative imaginary part because the bending modes are damped by a critical layer. The unstable mode remains the antisymmetric mode but its maximum growth rate is lower than unity. When the Froude number is increased above 1.83, the real part of the self-induction becomes negative, $\omega_{r}<0$ (equivalently $\mathscr{A}_{r}<0$ ), so that the antisymmetric mode (3.7) becomes stable whereas the symmetric mode (3.6) becomes unstable. The transition to a symmetric instability for $F_{h}>1.83$ is consistent with the existence in homogeneous fluids $\left(F_{h}=\infty, R o=\infty\right)$ of the Crow instability which is symmetric (Crow 1970). However, the theoretical maximum growth rate of the Crow instability in homogeneous fluids is $\sigma_{\max }=0.81$ for $\tilde{b}=8$ while the maximum growth rate for $1.83<F_{h}<8$ in figure $6(b)$ is lower, $\sigma_{\max }<0.5$, because of the critical layer, i.e. $\omega_{i}<0$.

For $F_{h} \gtrsim 3$, we see that the most amplified rescaled wavenumber $\hat{k}_{\text {max }} F_{h} b$ is proportional to $F_{h}$ (figure $6 a$ ). This is because the parameter $\mathscr{A}$ is mainly proportional to $1 / F_{h}^{2}$ when $F_{h} \gg 1$ (see Part 1 ). Thus, the most amplified wavenumber $\hat{k}_{\max }$ is almost independent of $F_{h}$ and inversely proportional to $b$ as for the Crow instability in homogeneous fluids (Crow 1970). However, it should be reminded that the expressions of the self-induction and mutual-induction functions used herein are valid only for $F_{h} \ll \tilde{b}^{2}$, i.e. $F_{h} \ll 64$ for the data plotted in figure 6 , and should be replaced for $F_{h} \rightarrow \infty$ by the functions valid in homogeneous fluids.

Figure $4(b)$ confirms that the displacements are antisymmetric for $F_{h}<1.83$ and symmetric for $F_{h}>1.83$. It can also be noticed that the displacements in the $x$ and $y$ directions are equal for $F_{h} \leqslant 1$ and then differ for $F_{h}>1$. This means that the vortices are not displaced along a direction making a $45^{\circ}$ angle with the $x$-axis when a critical layer exists.

Figures 7 and 8 show that the vertical vorticity of the most unstable eigenmodes predicted theoretically for $F_{h}=1.11$ and $F_{h}=4$ are in excellent agreement with the eigenmodes obtained numerically. The vertical vorticity of the eigenmode for $F_{h}=1.11$ 
(a)

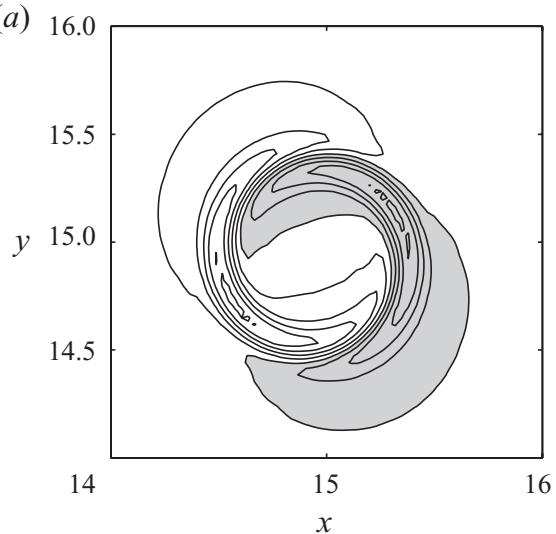

(b)

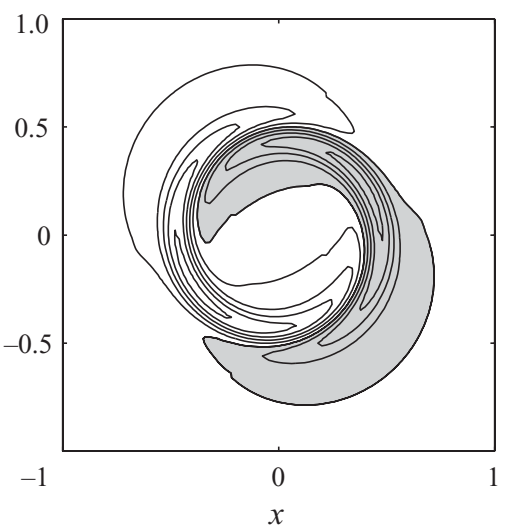

FIGURE 9. Comparison between the numerical $(a)$ and theoretical $(b)$ vertical velocity (imaginary part) of the most amplified eigenmode of two equal-strength counter-rotating vortices with $\tilde{b}=8$ for $F_{h}=1.11, R o=\infty, S c=1$ and $R e=50000$. Only a small region $2 R \times 2 R$ centred on the left vortex is displayed. The contour interval is 0.12 and the amplitude has been scaled with respect to the vertical vorticity (see text). Shaded areas are regions of positive values.

(figure 7) resembles the one for $F_{h}=0.1$ (figure 2) except for the presence of rapid variations at a fixed radius close to the vortex centres. This is due to the critical layer which occurs at the radius where the non-dimensional angular velocity of each vortex $\Omega$ is equal to $1 / F_{h}\left(r_{c}=0.46\right.$ for $\left.F_{h}=1.11\right)$. A critical layer is also present for $F_{h}=4$ but its amplitude is weaker so that it is almost not visible in figure 8 . Note that the theoretical vertical vorticity (figure $7 b$ ) takes into account not only the leading order terms as in (3.5) but also the first-order terms computed in Part 1.

The critical layer is more visible in the vertical velocity field displayed in figure 9 . Only a close view of size $2 R \times 2 R$ centred on the left vortex is shown because the vertical velocity of the perturbation is highly concentrated around the critical radius. The dimensional theoretical vertical velocity (figure $9 b$ ) for the vortex $(l)$ of the equal-strength counter-rotating vortex pair is given at leading order by (Part 1)

$$
\tilde{u}_{z}^{(l)}=\frac{\Gamma^{(l)}}{4 \pi} \hat{k} F_{h}^{2}\left[\left(-\bar{\Delta} x^{(l)}+\mathrm{i} \bar{\Delta} y^{(l)}\right) W \mathrm{e}^{\mathrm{i} \theta}+\left(\bar{\Delta} x^{(l)}+\mathrm{i} \bar{\Delta} y^{(l)}\right) W^{*} \mathrm{e}^{-\mathrm{i} \theta}\right],
$$

where $(r, \theta)$ are the cylindrical coordinates centred on the vortex $(l)$ (with $r$ nondimensionalized by $R$ ), the asterisk denotes the complex conjugate and

$$
\begin{aligned}
W= & \Omega^{2} r \frac{\Omega+R o^{-1}}{1-F_{h}^{2} \Omega^{2}}+\Omega_{c} r_{c} \frac{\Omega_{c}+R o^{-1}}{2 F_{h}{ }^{2} \Omega_{c}^{\prime}\left(r-r_{c}\right)} \\
& +\Omega_{c} r_{c} \pi \alpha \frac{\Omega_{c}+R o^{-1}}{2 F_{h}^{2} \Omega_{c}^{\prime}} \operatorname{Hi}\left[\alpha\left(r-r_{c}-\frac{\epsilon}{2 \Omega_{c}}\left(r_{c}-\frac{h_{c}}{r_{c}}\right) \cos 2 \theta-\epsilon \frac{\mathrm{i} \sigma}{\tilde{\Gamma} \Omega_{c}^{\prime}}\right)\right],
\end{aligned}
$$

where the subscript $c$ indicates the value taken at $r_{c}, \alpha=-\mathrm{i} \operatorname{Re}^{1 / 3} \operatorname{sgn}\left(\Gamma^{(l)}\right)\left(2 \Omega_{c}^{\prime} /\right.$ $(1+1 / S c))^{1 / 3}, \epsilon=\tilde{\Gamma} / \tilde{b}^{2}$ is the non-dimensional strain, Hi is the Scorer's function (Abramowitz \& Stegun 1965) and $h(r)$ is a function describing the elliptical shape of the vortex (see Part 1). As explained in Part 1, the solution (3.10) is a composite approximation between the inviscid solution (first term of the right-hand side) and the solution valid inside the critical layer (last term of the right-hand side). The latter solution smoothes the singularity by taking into account higher-order effects, namely 

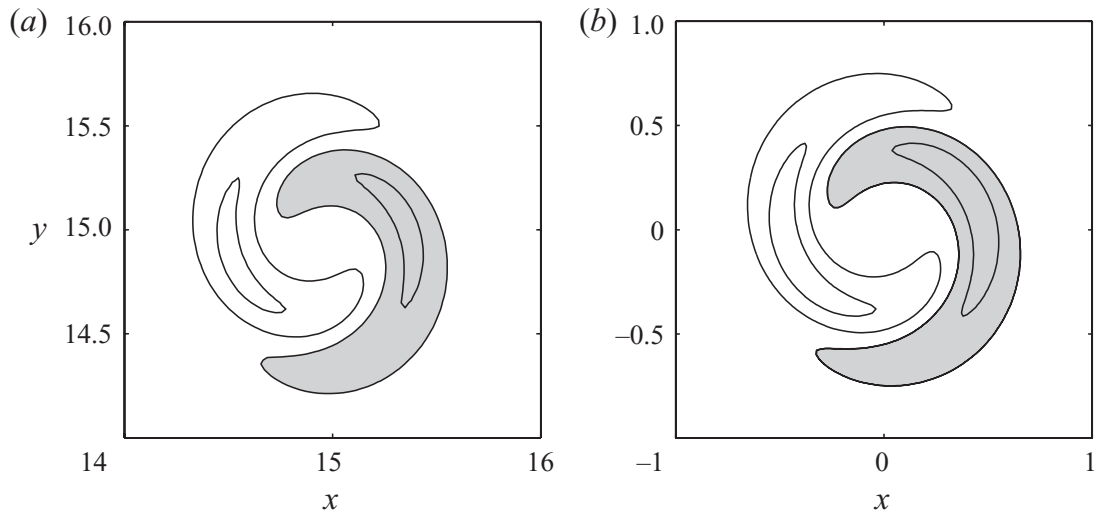

FIGURE 10. Similar to figure 9 but for $R e=5000$ and $\hat{k} F_{h} b=1.13$.
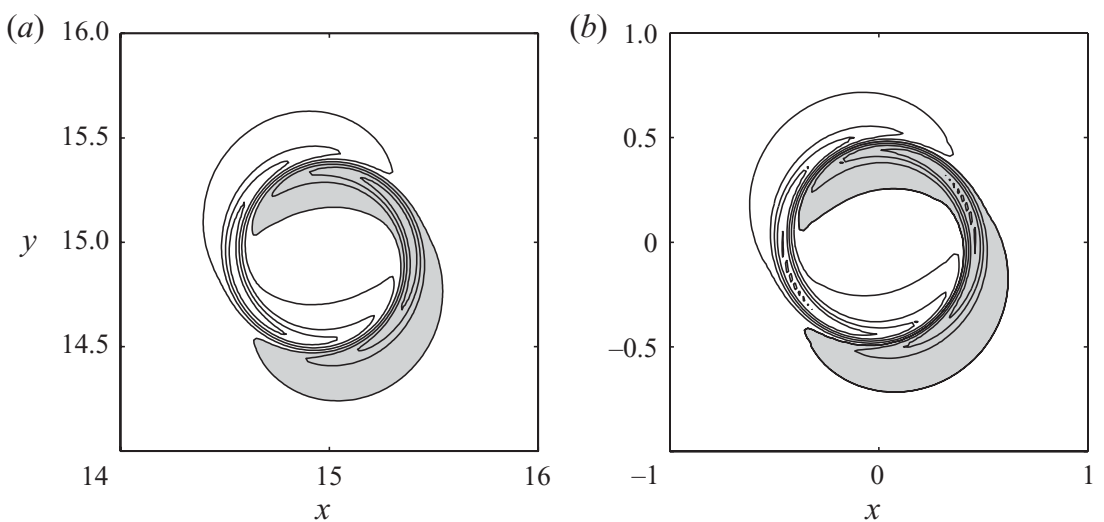

FiguRE 11. Similar to figure 9 but for $R e=250000$ and $\hat{k} F_{h} b=1.13$.

the viscous effects which scale like $R e^{1 / 3}$, the ellipticity of the basic vortex (middle term of the argument of the function $\mathrm{Hi}$ ) and the growth of the disturbances (last term of the argument of $\mathrm{Hi}$ ), both of which scale like the non-dimensional strain $\epsilon$. The relative importance of the latter effects thus depends on the parameter $R e \epsilon^{3}$. We see in figure $9(b)$ that the theoretical vertical velocity field (3.9) is in excellent agreement with the vertical velocity field obtained numerically (figure $9 a$ ) The agreement is not only qualitative but also quantitative since, in each case, the velocity fields of the perturbation have been normalized the same way by imposing that the squared vertical vorticity integrated over the domain is unity, i.e. $\int \tilde{\zeta}^{2} \mathrm{~d} x \mathrm{~d} y=1$.

The dependence of the theoretical solution with respect to the Reynolds number $R e$ is further tested in figure 10 for $R e=5000$ and figure 11 for $R e=250000$. (The resolution has been increased to $1280 \times 1024$ for this Reynolds number.) For both $R e$, the amplitude and pattern of the modes predicted theoretically and numerically are in remarkable agreement. For $R e=5000$, we see that the vertical velocity has lower extremum values and exhibits smoother variations around the critical radius than for $R e=50000$. In this case, the parameter $R e \epsilon^{3}$ is small and equal to -0.02 . Thus, the terms of order $\epsilon$ are negligible and the critical layer is almost a purely viscous critical layer with a typical amplitude of the vertical velocity proportional to $R e^{1 / 3}$. The corresponding radial length scale, which captures the variations of the critical 

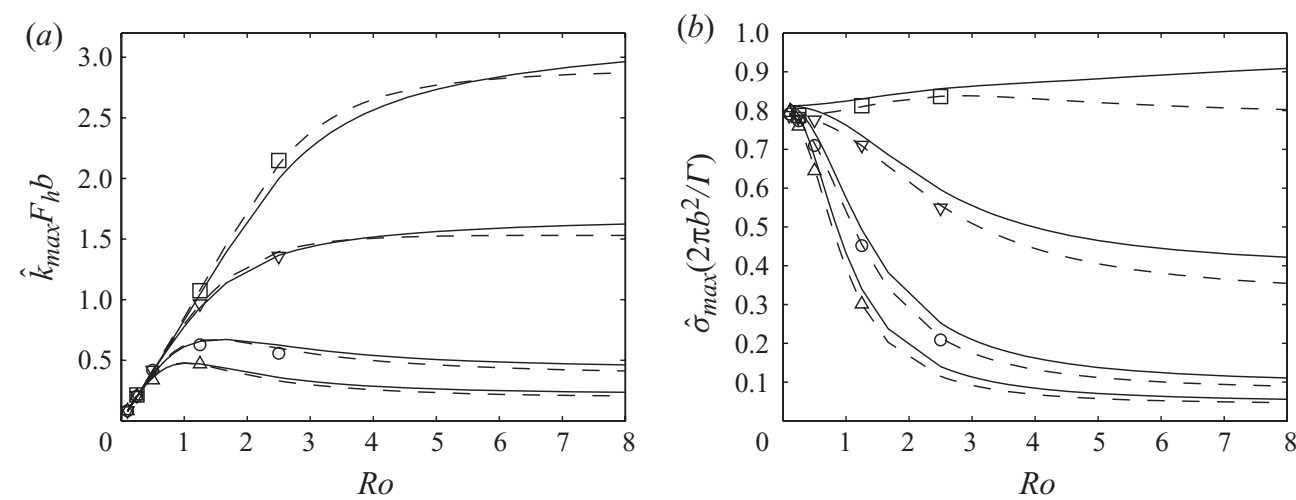

FIGURE 12. Most amplified vertical wavenumber $(a)$ and the maximum growth rate $(b)$ as a function of the Rossby number for two equal-strength counter-rotating Lamb-Oseen vortices for $F_{h}=0.1, \tilde{b}=8, S c=1$ and different Reynolds numbers (going from top to bottom): $R e=50000(\square), R e=1000(\nabla), R e=200(\bigcirc)$ and $R e=100(\triangle)$. The solid lines show the theoretical predictions and the symbols show the results of a direct numerical stability analysis. The dashed lines show improved theoretical predictions taking into account the periodic boundary conditions used in the direct numerical stability analysis.

layer around the critical radius, scales like $R e^{-1 / 3}$. For $R e=250000$ (figure 11), the amplitude of the vertical velocity is larger and more concentrated around the critical radius than for $R e=50000$. In this case, we have $R e \epsilon^{3}=-0.95$ so that the $O(\epsilon)$ effects are not negligible. The term involving the exponential growth of the perturbation in (3.10) has a significant effect. As shown in Part 1, it makes the amplitude of the vertical velocity field in the critical layer scaling like $1 /|\epsilon|$ instead of $R e^{1 / 3}$. Correspondingly, the width of the critical layer is of order $|\epsilon|$ instead of $1 / R e^{1 / 3}$. Thereby, the critical layer does not become infinitely thin when $R e$ tends to infinity but saturates to a size of order $|\epsilon|$. For this reason, the critical layer in figure 11 is in fact thicker than a critical layer for the same Reynolds number and $\epsilon=0$ (not shown). In contrast, the elliptical shape of the critical layer described by the middle term of the argument of $\mathrm{Hi}$ in (3.10) is not visible in figure 11.

\subsubsection{Effect of the Reynolds number}

So far, only very high Reynolds numbers close to the inviscid limit have been studied. Figure 12 now shows the effect of the Reynolds number on the most amplified wavenumber and maximum growth rate for a fixed low Froude number $F_{h}=0.1$, i.e. when there is no critical layer. The theoretical results (solid lines) have been obtained by means of the viscous self-induction function (2.15). We see that the agreement with the numerical results is very good even for the lowest Reynolds number $R e=100$. As shown by the dashed lines, an even better agreement can be achieved by taking into account in the theory the effect of the periodic boundary conditions used in the numerical stability analysis (see the Appendix).

The magnitude of dissipative effects strongly depends on the Rossby number. For $R o=\infty$, the zigzag instability is strongly damped even for relatively large Reynolds number values: for example, the growth rate for $R e=1000$ is reduced by $60 \%$ compared to its inviscid value (figure $12 b$ ). The corresponding most unstable wavenumber also decreases (figure 12a). Viscous effects are high because the most amplified wavenumber $\hat{k}_{\max }$ is inversely proportional to $F_{h}$. The vertical viscous dissipation thus scales like $\hat{k}_{\max }^{2} / R e \propto 1 /\left(R e F_{h}^{2}\right)$ and is large when $F_{h}$ is small. The 
magnitude of viscous effects decreases with $R o$ and for $R o=0.25$, the growth rate for $R e=100$ is only slightly smaller than for $R e=50000$. This is mainly due to the decrease of the most amplified wavenumber with $R o$ but also partly due to the decrease of the coefficient $\mathscr{V}$ (see (2.15)) with $R o$. For smaller $R o$, the coefficient $\mathscr{V}$ even becomes negative, i.e. the viscous effects become destabilizing. Thus, the growth rate for $R o=0.1$ is slightly larger for $R e=100$ than for $R e=50000$. The difference is nevertheless very small and cannot be seen with the naked eye in figure $12(b)$.

\subsection{Stability of an equal-strength co-rotating vortex pair}

In this section, the theory is compared to the results of the numerical stability analysis of an equal-strength co-rotating pair of Lamb-Oseen vortices performed by Otheguy et al. $(2006 a, b)$ in a stratified and rotating fluid. Note that the Froude and Rossby numbers defined in Otheguy et al. $(2006 a, b)$ are twice those used herein (see (2.1)).

When the two vortices have the same circulation, i.e. $\tilde{\Gamma}=1$, both their Froude numbers and Rossby numbers are equal: $F_{h}^{(l)}=F_{h}^{(r)} \equiv F_{h}$ and $R o^{(l)}=R o^{(r)} \equiv R o$, implying that their self-inductions are always equal, i.e. $\omega^{(l)}=\omega^{(r)} \equiv \omega$. Like in homogeneous fluids (Jimenez 1975), the dispersion relation (2.17) thus decouples between symmetric and antisymmetric eigenvectors:

$$
\begin{aligned}
& \overline{\boldsymbol{\Delta}} \boldsymbol{x}_{s}=\frac{1}{2}\left[\bar{\Delta} x_{s}, \bar{\Delta} y_{s},-\bar{\Delta} x_{s},-\bar{\Delta} y_{s}\right], \\
& \overline{\boldsymbol{\Delta}} \boldsymbol{x}_{a}=\frac{1}{2}\left[\bar{\Delta} x_{a}, \bar{\Delta} y_{a}, \bar{\Delta} x_{a}, \bar{\Delta} y_{a}\right],
\end{aligned}
$$

where $\bar{\Delta} x_{s}=\bar{\Delta} x^{(l)}-\bar{\Delta} x^{(r)}, \bar{\Delta} y_{s}=\bar{\Delta} y^{(l)}-\bar{\Delta} y^{(r)}, \bar{\Delta} x_{a}=\bar{\Delta} x^{(l)}+\bar{\Delta} x^{(r)}$ and $\bar{\Delta} y_{a}=\bar{\Delta} y^{(l)}+$ $\bar{\Delta} y^{(r)}$. The definition of these modes is not the same as in $\S 3.1$ because the symmetry of the basic state is different: equal-strength co-rotating vortex pairs have a central symmetry with respect to the mid-point between the vortex centres. As before, the symmetric mode is the mode which preserves this symmetry of the basic state. The growth rates of symmetric and antisymmetric modes are given by

$$
\begin{aligned}
& \left(\sigma_{s}-\tilde{b}^{2} \omega_{i}\right)^{2}=\left(3-\tilde{b}^{2} \omega_{r}+\chi\right)\left(\tilde{b}^{2} \omega_{r}-1+\Psi\right), \\
& \left(\sigma_{a}-\tilde{b}^{2} \omega_{i}\right)^{2}=\left(3-\tilde{b}^{2} \omega_{r}-\chi\right)\left(\tilde{b}^{2} \omega_{r}-1-\Psi\right) .
\end{aligned}
$$

It can be easily checked that the growth rate of the symmetric mode (3.13) in the limits $F_{h}=0, R o=\infty$ and $R e=\infty$ agrees with the asymptotic result obtained by Otheguy et al. (2007) for a strongly stratified and non-rotating inviscid fluid.

\subsubsection{Effect of the Rossby number for a strongly stratified fluid}

The growth rate of the symmetric mode (3.13) is plotted in figure 13 as a function of the rescaled vertical wavenumber $\hat{k} b F_{h}$ for various Rossby numbers for $\tilde{b}=6.7$ and a fixed Froude number $F_{h}=0.5$. As found by Otheguy et al. (2006a), we see that the symmetric mode is unstable for all the Ro values while it is known to be stable in homogeneous fluids (Jimenez 1975). The antisymmetric mode (3.12) always remains stable in stratified-rotating fluids like in homogeneous fluids.

As seen in figure 13, the theoretical growth rate (3.13) is in good agreement with the numerical results of Otheguy et al. (2006a) except for the intermediate negative values of the Rossby number: $R o=-2.5$ and $R o=-1.25$ (figure $13 b$ ). For these values, the theory significantly underestimates the growth rate. We can also notice that there is a difference between the theory and numerics for small wavenumbers: the numerical growth rate does not seem to tend to zero for zero wavenumber contrary to the theoretical growth rate. As shown in figure 22(b) in the Appendix, this difference comes from the use of periodic boundary conditions in the numerical stability analysis of Otheguy et al. $(2006 a)$. 

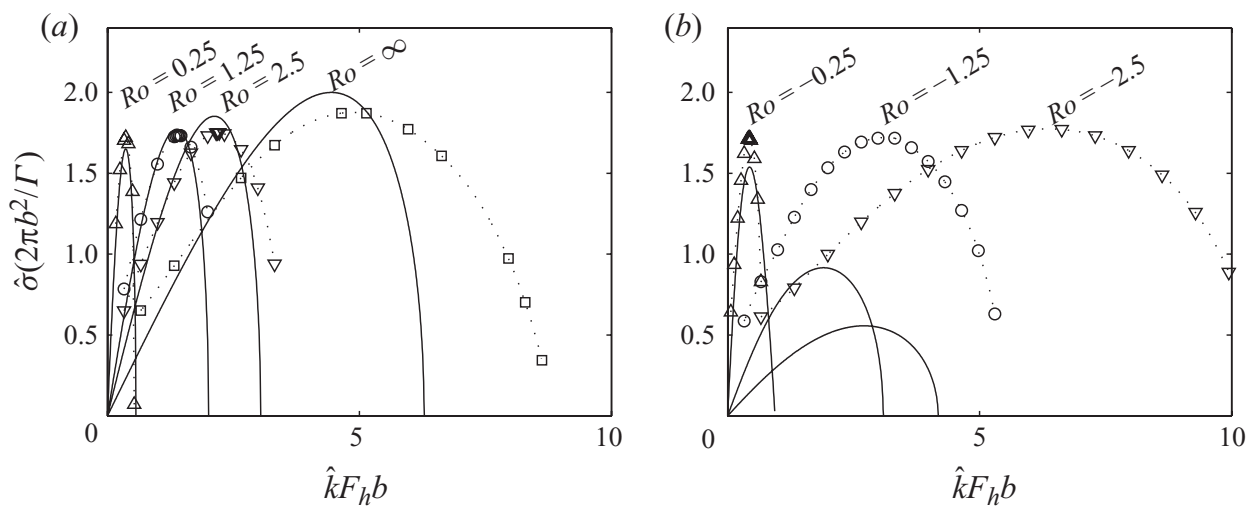

FIGURE 13. Growth rate of the zigzag instability as a function of the rescaled vertical wavenumber $\hat{k} F_{h} b$ for two equal-strength co-rotating Lamb-Oseen vortices with $\tilde{b}=6.7$ for $F_{h}=0.5, R e=8000$ and for cyclonic $(a)$ and anticyclonic $(b)$ rotations. The solid lines (-) show the theoretical growth rate (3.13) and the symbols connected by dotted lines show the numerical results of Otheguy et al. (2006a) for $R o=\infty(\square), R o=2.5(\nabla), R o=1.25(\bigcirc)$, $R o=0.25(\triangle)$ in $(a)$ and $R o=-2.5(\nabla), R o=-1.25(\bigcirc), R o=-0.25(\triangle)$ in $(b)$.
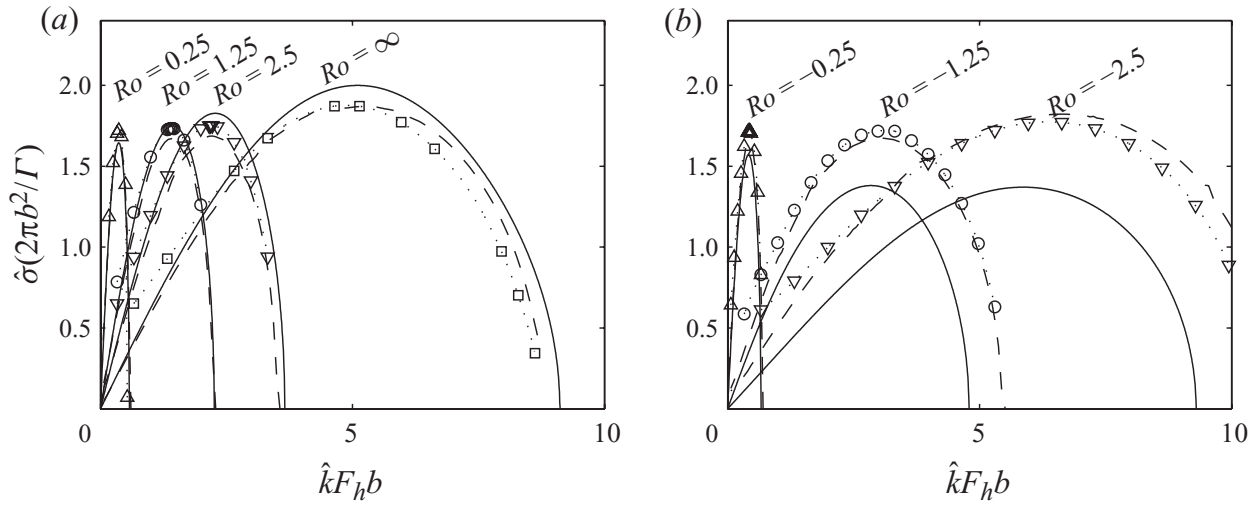

FIGURE 14. Similar to figure 13 except that the self-induction function has been replaced by the frequency of the bending modes calculated numerically in the theoretical growth rate (3.13). The results of the combined asymptotical-numerical model developed in Appendix E of Part 1 is shown by dashed lines.

Since the theory works well for the positive values of $R o$ (figure 13a), the reason for the large discrepancy for $R o=-2.5$ and $R o=-1.25$ should be sought in the parameter $\delta\left(F_{h}, R o\right)$ of the self-induction function since it is the only term in (3.13) which depends on the sign of $R o$. The discrepancy between the theory and the numerics is maximum around $R o \approx-2$, and this corresponds to the Rossby number for which $\delta$ is minimum for a given $F_{h}$. This means that $R o \approx-2$ corresponds to the Rossby numbers where the self-induction function increases most slowly with $\hat{\beta}$. Thus, the relative influence of the neglected order $O\left(\hat{\beta}^{4}\right)$ in the self-induction $\omega$ is expected to be higher around $R o \approx-2$. This hypothesis has been tested by plotting in figure 14 the theoretical growth rate (3.13) with the self-induction $\omega$ replaced by the exact frequency $\omega_{e}$ of the slow bending modes obtained numerically in Part 1 . This is equivalent to taking into account higher-order terms of $\hat{\beta}$ in the self-induction function. We see that the agreement improves considerably for $R o=-2.5$ and $R o=-1.25$ although it 
is still not as good as for the other Rossby numbers. There is also an improvement at large wavenumbers for the other values of $R o$, especially $R o=\infty$ and $R o=2.5$ (figure 14). Furthermore, the spatial structure of the bending waves at higher orders in vertical wavenumber can also be taken into account by using the eigenmode of a single vortex computed numerically for finite $\hat{k}$. However, this is not straightforward since it also requires to compute asymptotically for finite wavenumber the interaction between these waves and the strain and the mutual-induction effects. Such analysis is carried out in Appendix E of Part 1. As shown by the dashed lines in figure 14, the predictions of this combined numerical-asymptotical analysis fully agree with the results of the direct numerical stability analysis for all the Rossby numbers.

The reason for the significant improvement of the theoretical growth rate when the self-induction function is replaced by $\omega_{e}$ can be further explained by using the fact that the mutual-induction functions are bounded: $0 \leqslant \Psi \leqslant 1$ and $0 \leqslant \chi \leqslant 1.06$ (see (2.7) and (2.8)). Therefore, an upper bound for the growth rate (3.13) of the symmetric mode is given by

$$
\sigma_{s}^{2} \leqslant\left(4.06-\tilde{b}^{2} \omega_{r}\right) \tilde{b}^{2} \omega_{r}
$$

because $\omega_{i} \leqslant 0$. Since the bound is a parabolic function of the single variable $\tilde{b}^{2} \omega_{r}$, the overall upper bound for the maximum growth rate is $\sigma_{\text {smax }}=2.03$ and is reached when $\omega_{r}=\omega_{m} \equiv 2.03 / \tilde{b}^{2}$. Therefore, when $\omega_{r}$ increases monotonically with $\hat{k}$ at least up to $\omega_{m}$, the overall upper bound can be reached potentially. However, when $\omega_{r}$ is not a monotonic function of $\hat{k}$ but has a maximum $\omega_{\text {rmax }}$, which is lower than $\omega_{m}$, the upper bound for the maximum growth rate is reduced to

$$
\sigma_{\operatorname{smax}}=\sqrt{\left(4.06-\tilde{b}^{2} \omega_{\text {rmax }}\right) \tilde{b}^{2} \omega_{\text {rmax }}}
$$

The latter case precisely corresponds to what happens for $R o=-2.5$ and $R o=-1.25$ : for the separation ratio $\tilde{b}=6.7$, we have $\omega_{m}=0.045$ whereas the maximum of the self-induction function is lower: $\omega_{\text {rmax }} \approx 0.015-0.02$ (see figure 7 of Part 1 ). Thus, the upper bound is only $\sigma_{\text {smax }} \approx 1.5$. In contrast, the overall upper bound, $\sigma_{\text {smax }}=2.03$, is not out of reach for all the other $R o$ values because $\omega_{\text {rmax }} \geqslant 0.045$ (see figure 7 of Part 1). This is the reason why the theoretical maximum growth rate is lower for $R o=-2.5$ and $R o=-1.25$ and not for the other values of $R o$ for $\tilde{b}=6.7$. There is a large improvement when the exact frequency $\omega_{e}$ of the slow bending modes is used since it always increases monotonically with $\hat{k}$ even for $R o=-2.5$ and $R o=-1.25$.

In practice, the theoretical growth rate is expected to be quantitatively valid at least in the range of wavenumbers where the self-induction function correctly approximates the frequency of the bending modes. As shown in figure 7 of Part 1, this range is $0<\hat{k} R F_{h}<0.2-0.3$ for $|R o|=1.25$ and $|R o|=2.5$. For $\widetilde{b}=6.7$, this range corresponds to only a small range in terms of $\hat{k} b F_{h}: 0<\hat{k} b F_{h}<1.5-2$. This is an additional reason for the discrepancies for $R o=-1.25$ and $R o=-2.5$ since the numerical growth rate extends to wavenumbers outside this range (up to $\hat{k} b F_{h} \approx 5$ and $\hat{k} b F_{h} \approx 11$, respectively) and much larger than for the corresponding positive values of $R o$ (up to $\hat{k} b F_{h} \approx 2$ and $\hat{k} b F_{h} \approx 3$ for $R o=1.25$ and $R o=2.5$, respectively).

From the reasoning above, the range of rescaled wavenumbers $\hat{k} b F_{h}$ where the theoretical growth rate should be quantitatively valid is expected to increase when $\tilde{b}$ is larger. This is confirmed in figure 15 where the theoretical growth rate for $\tilde{b}=15$ is compared to the results of a direct numerical stability analysis using the same method as in Otheguy et al. (2006b). We see that the agreement is excellent even for $R o=-1.25$ and $R o=-2.5$. There is still a discrepancy at large wavenumbers, $\hat{k} b F_{h}>5$, for the latter $R o$ value but this is not surprising since the wavenumber range 

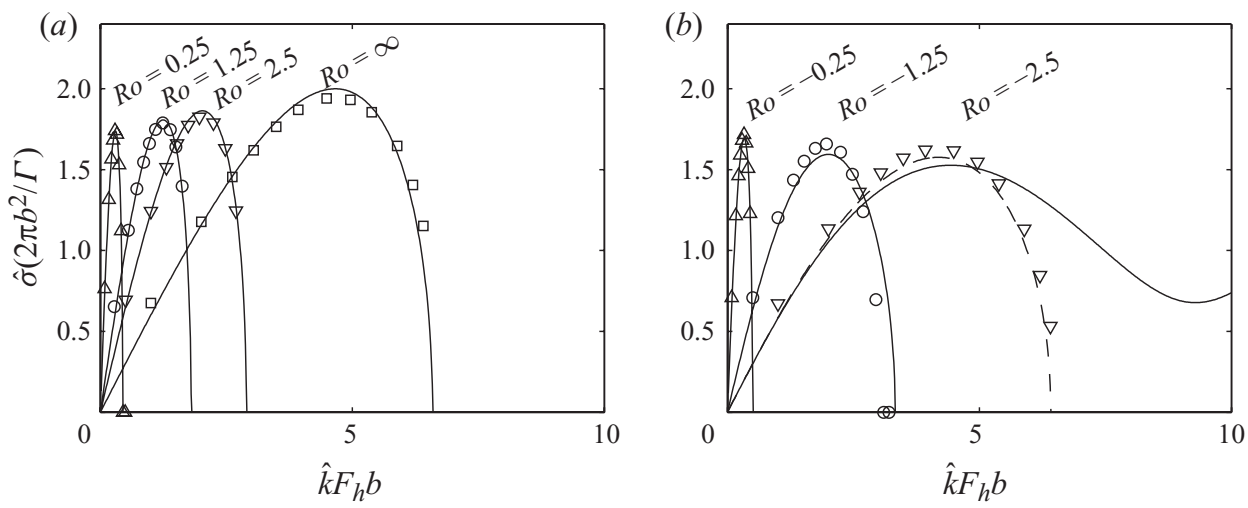

FIGURE 15. Similar to figure 13 except that $\tilde{b}=15, R e=50000$, and $F_{h}=0.1$. The dashed line in $(b)$ shows the theoretical growth rate for $R o=-2.5$ with the self-induction function replaced by the frequency $\omega_{e}$ of the bending modes computed numerically.
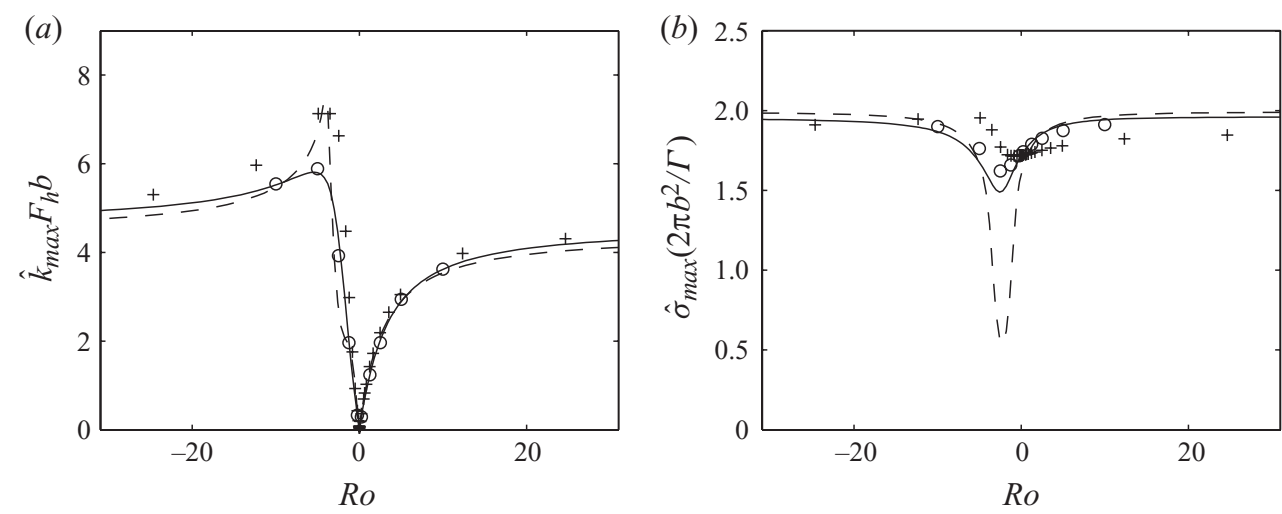

FIGURE 16. Most amplified vertical wavenumber $(a)$ and maximum growth rate $(b)$ of the zigzag instability of two equal-strength co-rotating Lamb-Oseen vortices as a function of the Rossby number. The curves show the theoretical predictions and the symbols show the results of direct numerical stability analyses for $\tilde{b}=6.7, F_{h}=0.5, R e=8000(---$ and +$)$ and $\tilde{b}=15$, $F_{h}=0.1, R e=50000$ ( $\square$ and $O$ ).

where the self-induction function is quantitatively valid is $0<\hat{k} b F_{h}<4.5$ for $\tilde{b}=15$. The agreement becomes almost perfect when the self-induction function is replaced by the frequency $\omega_{e}$ of the slow bending modes computed numerically (dashed line in figure $15 b$ ). We can also notice that there is no discrepancy between the numerical and theoretical growth rates for low wavenumbers in figure 15 in contrast to figures 13 and 14. This is because we have used a larger computational domain $(75 R \times 60 R)$ than in Otheguy et al. $(2006 a)(30 R \times 30 R)$ in order to reduce the effects of the periodic boundary conditions.

Figure 16(a) shows a further comparison of the most amplified wavenumber as a function of the Rossby number between the theoretical predictions and the numerical results for $\tilde{b}=6.7$ (Otheguy et al. 2006a) and $\tilde{b}=15$. We see that the agreement is very good for both values of $\tilde{b}$. Like for a counter-rotating vortex pair, the most amplified wavenumber $\hat{k}_{\max } F_{h} b$ is constant for large Rossby number whereas it scales like $R o$ for small $R o$, in agreement with the quasi-geostrophic theory. In between, $\hat{k}_{\max } F_{h} b$ increases monotonically with the Rossby number for positive values of $R o$ while it increases and then decreases with $|R o|$ for negative values of $R o$. This figure also 

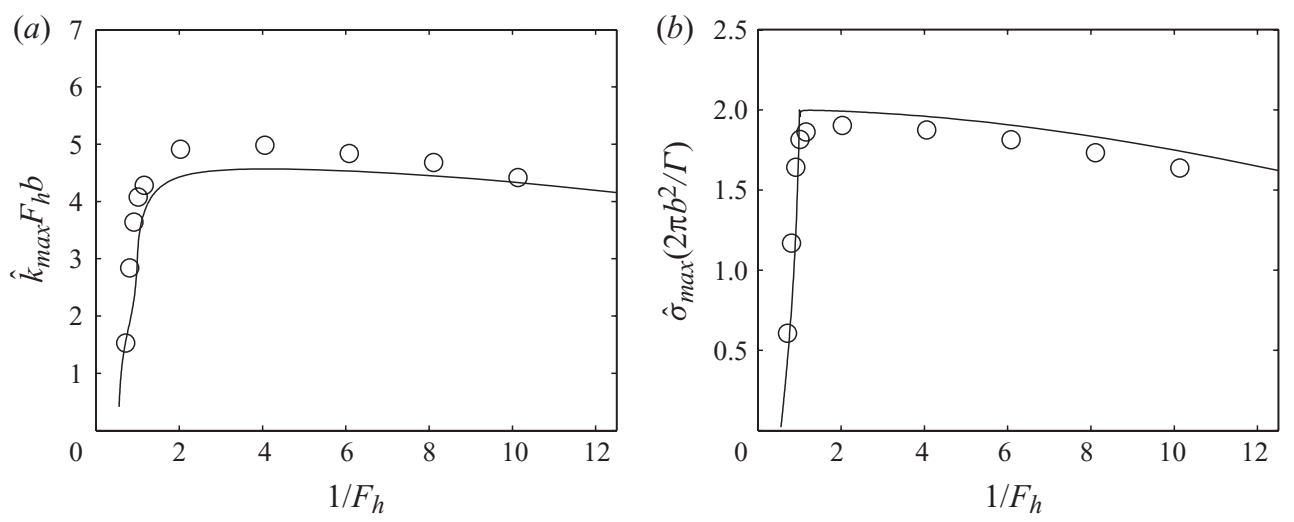

Figure 17. Most amplified vertical wavenumber $(a)$ and maximum growth rate $(b)$ of the zigzag instability of two equal-strength co-rotating Lamb-Oseen vortices as a function of the inverse of the Froude number for $R o=\infty, R e=8000$ and $\tilde{b}=6.7$. The solid line shows the theoretical prediction and the symbols $(\bigcirc)$ show the results of the direct numerical stability analysis of Otheguy et al. (2006b). Note that the theoretical results are independent of $\tilde{b}$ when $R o=\infty$.

shows that the values $R o=-2.5$ and $R o=-1.25$ are right in the region where the steepest variation of $\hat{k}_{\max }$ is observed. Thus, even if there is a large discrepancy between the theory and the numerics for these Rossby numbers for $\vec{b}=6.7$ (as seen in figure 13), this discrepancy is almost not visible in figure 16(a).

The corresponding maximum growth rate is also plotted in figure $16(b)$ as a function of $R o$. The anomalous decrease of the theoretical growth rate for $\tilde{b}=6.7$ (dashed line) around $R o \approx-2$ is clearly visible in this figure. However, the agreement between the theoretical and the numerical results for $\tilde{b}=6.7$ is very good outside this region. There is also a small decrease of the theoretical growth rate for $\tilde{b}=15$ around $R o=-2$, but in this case, a similar behaviour is observed in the numerical results and the agreement is always excellent whatever the Rossby number.

\subsubsection{Effect of the Froude number for a non-rotating fluid}

When $R o=\infty$, the growth rates (3.13) and (3.14) of the symmetric and antisymmetric modes simplify to

$$
\begin{aligned}
& \left(\sigma_{s}-\tilde{b}^{2} \omega_{i}\right)^{2}=\left(4-\tilde{b}^{2} \omega_{r}\right) \tilde{b}^{2} \omega_{r}, \\
& \left(\sigma_{a}-\tilde{b}^{2} \omega_{i}\right)^{2}=-\left(2-\tilde{b}^{2} \omega_{r}\right)^{2} .
\end{aligned}
$$

We directly see in (3.17) that the antisymmetric mode can never be unstable. In contrast, the growth rate of the symmetric mode (3.16) can be positive if $0<\tilde{b}^{2} \omega_{r}<4$. When this is the case (i.e. $\mathscr{A}_{r}>0$ ), the maximum growth rate and the rescaled most amplified wavenumber are similar to those derived for an equal-strength counterrotating vortex pair:

$$
\sigma_{\text {max }}=2 \frac{|\mathscr{A}|+\mathscr{A}_{i}}{\left|\mathscr{A}_{r}\right|}, \quad \hat{k}_{\text {max }} F_{h} b=2 \sqrt{\frac{1}{\left|\mathscr{A}_{r}\right|}} \sqrt{1-\frac{\left|\mathscr{A}_{i}\right|}{|\mathscr{A}|}} .
$$

They are plotted as a function of the inverse Froude number $1 / F_{h}$ (solid lines) in figure 17. Like for an equal-strength counter-rotating vortex pair (\$3.1), the maximum growth rate (figure $17 b$ ) is independent of the Froude number for $1 / F_{h}>1$ (except for a slight decrease as $F_{h} \rightarrow 0$ due to viscous effects) and drops abruptly to zero when 
$1 / F_{h}<1$. However, unlike equal-strength counter-rotating vortex pairs, the maximum growth rate remains zero for $1 / F_{h}<0.55$ (i.e. $F_{h}>1.83$ ), meaning that the vortex pair is stable to any long-wavelength bending perturbations. The rescaled most amplified wavenumber $\hat{k}_{\max } F_{h} b$ (figure $17 a$ ) is also almost constant when $1 / F_{h}>1$ and decreases abruptly when $1 / F_{h}<1$. The theoretical results are in very good agreement with the numerical results (symbols) of the direct numerical stability analysis of Otheguy et al. (2006b). As already mentioned, the sudden stabilization for $F_{h}>1$ is due to the presence of a critical layer which damps the bending modes of each vortex. The threshold of complete stabilization, $F_{h}=1.83$, corresponds to the Froude number above which the real part of the self-induction function $\omega_{r}$ becomes negative for $R o=\infty$. Note that the condition of validity of the theory, $F_{h} \ll \tilde{b}^{2}=44$ for $\tilde{b}=6.7$, is still fully satisfied when $F_{h}=1.83$.

\subsection{Combined effects of the Froude and Rossby numbers}

To give a complete overview of the stability properties of equal-strength counterrotating and co-rotating pairs of Lamb-Oseen vortices, the contours of maximum theoretical growth rate are plotted in the parameter space Froude-Rossby numbers in figures $18(a)$ and $18(b)$, respectively. In both cases, the maximum growth rate is highest and approximately constant in the domain $F_{h} \leqslant 1$ : it is equal to the strain for a counter-rotating vortex pair and twice the strain for a co-rotating vortex pair with a weak dependence on $R o$. An abrupt decrease of the maximum growth rate occurs for $F_{h}>1$ because the critical layer damps the slow bending modes. The steepness of this decrease, however, depends on $R o$ : it is very steep for large $|R o|$ and smooth when $R o \rightarrow 0$. In the case of a co-rotating vortex pair (figure 18b), the maximum growth rate vanishes when $F_{h} \gtrsim F_{h c}(R o)$, where $F_{h c}$ is a critical Froude number depending mainly on the Rossby number and such that $F_{h c} \sim 1.8$ for $R o \gg 1$ and $F_{h c} \gg 1$ for $R o \ll 1$. However, it must be emphasized that the location of the threshold for small $R o$ cannot be determined from the present theory since $F_{h c}$ is located beyond the domain of validity of the theory: $F_{h} \ll \tilde{b}^{2}$. In addition, note that $F_{h c}$ depends weakly on $\tilde{b}$ for finite $R o$. In the case of a counter-rotating vortex pair (figure $18 a$ ), the maximum growth rate for large $R o$ re-increases slowly when $F_{h} \gtrsim 1.5-1.8$. The most unstable eigenmode is always purely symmetric for a co-rotating vortex pair. For a counter-rotating vortex pair (figure 18a), the most unstable eigenmode has no exact symmetry except in the limits: $\left(R o \rightarrow 0, F_{h} \ll \tilde{b}^{2}\right)$ or $\left(R o \rightarrow \infty, F_{h}<1.83\right)$, where it is purely antisymmetric, and in the limit $\left(R o \rightarrow \infty, F_{h}>1.83\right)$, where it is purely symmetric. In the rest of the parameter space, the instability is quasi-antisymmetric in the shaded region and quasi-symmetric in the white region.

\subsection{Unequal-strength vortex pairs}

We finish by investigating the stability of unequal-strength vortex pairs. However, the purpose of this section is not to comprehensively describe the stability characteristics for arbitrary circulation ratio $\tilde{\Gamma}$ but only to qualitatively illustrate the main differences from the cases of equal-strength vortex pairs, i.e. $\tilde{\Gamma}= \pm 1$.

As a typical example, figure 19 shows the growth rate for various circulation ratios $\tilde{\Gamma}$ in the case of a strongly stratified and rotating fluid: $F_{h}^{(l)}=0.5$ and $R o^{(l)}=5$. The most striking difference from equal-strength vortex pairs is the presence of several peaks. For co-rotating vortex pairs (figure 19a), the growth-rate curves always start at $\hat{k}=0$ but they exhibit two distinct peaks for $\tilde{\Gamma}=0.6$ and three for $\tilde{\Gamma}=0.4$. In each case, the eigenmode corresponding to the first peak is quasi-symmetric (for example, $\bar{\Delta} \boldsymbol{x}=(0.39,-0.35,-0.15,0.84)$ for $\tilde{\Gamma}=0.4)$ and can be traced back to the 

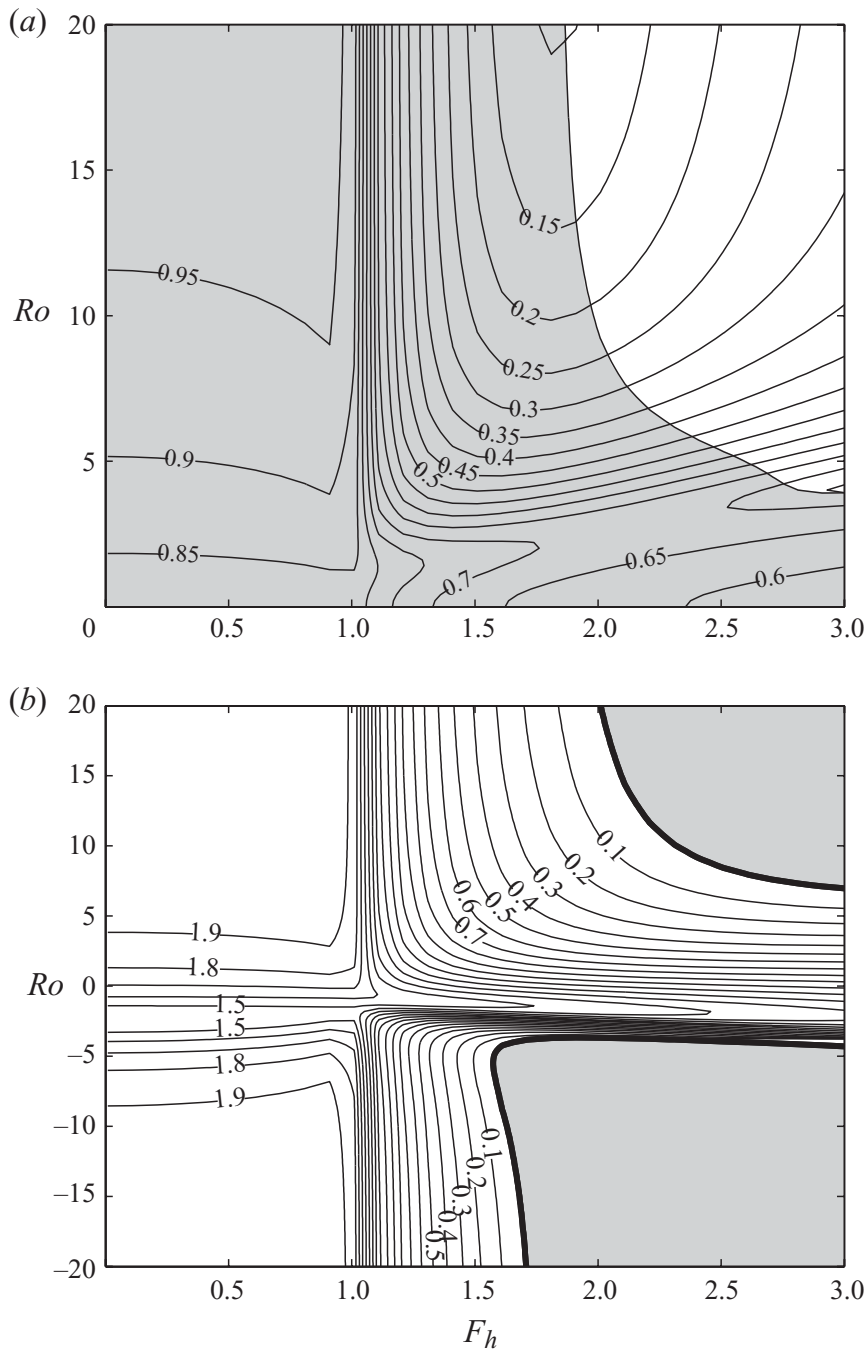

FIGURE 18. Contours of maximum growth rate $\hat{\sigma}_{\max } 2 \pi b^{2} / \Gamma$ as a function of the Froude number $F_{h}$ and Rossby number $R o$ for $(a)$ a counter-rotating vortex pair and $(b)$ a co-rotating vortex pair for $\tilde{b}=12$ and $R e=\infty$. In $(a)$, the eigenmode is mostly antisymmetric in the shaded domain and mostly symmetric in the unshaded region. In $(b)$, the stable domain in the long-wavelength limit is light shaded and delimited by bold lines (see $\S 4$ ). The contour interval is 0.05 in $(a)$ and 0.1 in $(b)$.

symmetric instability existing for $\tilde{\Gamma}=1$. We see that the maximum growth rate of this instability markedly decreases when $\tilde{\Gamma}$ decreases. The intermediate peak for $\tilde{\Gamma}=0.4$ is much lower and has a non-zero frequency (not shown) in contrast to the other two. The maximum growth rate of the last peak is approximately unity regardless of $\tilde{\Gamma}$. Remarkably, this peak is higher than the first one for small $\tilde{\Gamma}$, as seen for $\tilde{\Gamma}=0.4$ in figure $19(a)$. The associated most amplified wavenumber increases as the circulation ratio $\tilde{\Gamma}$ decreases so that the third unstable band separates from the others for small $\tilde{\Gamma}$. The corresponding most unstable eigenmode is a displacement of the weak vortex while the strong vortex barely moves (for example, $\bar{\Delta} x=(-0.04,0.01,-0.69,0.72)$ for $\tilde{\Gamma}=0.4)$. Therefore, this instability can be interpreted as a drift of the weak vortex in 

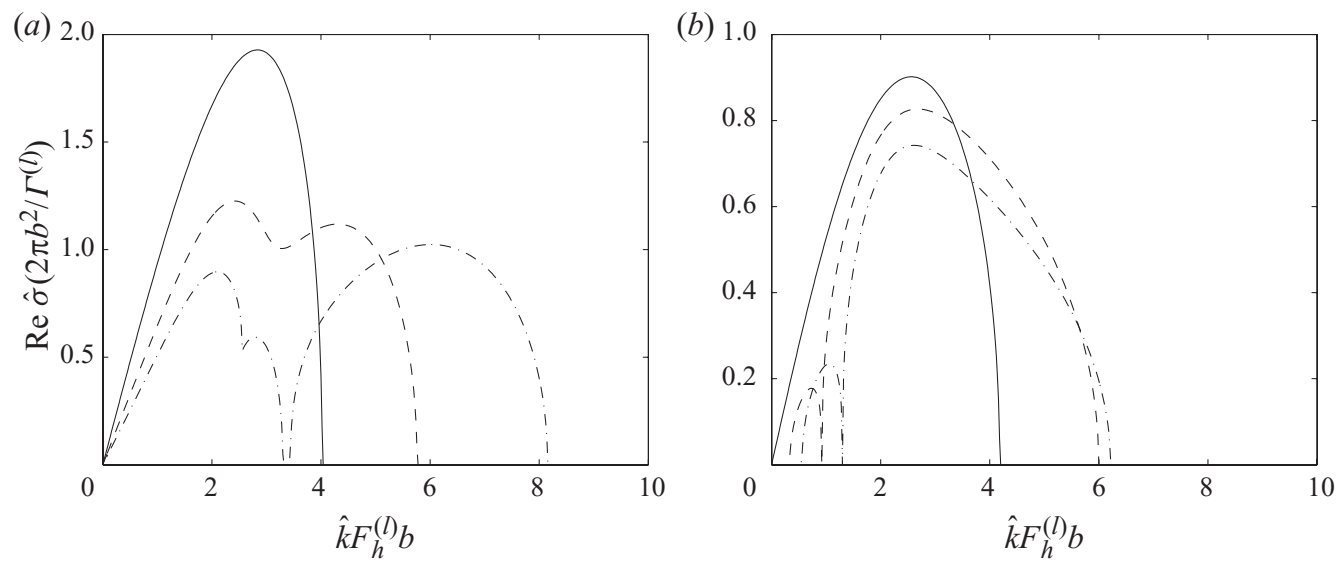

FIGURE 19. Growth rate (real part) of unequal-strength vortex pairs as a function of the rescaled vertical wavenumber $\hat{k} F_{h}^{(l)} b$ for $\tilde{b}=12, F_{h}^{(l)}=0.5, R o^{(l)}=5, R e^{(l)}=\infty$ and for different positive $(a)$ and negative $(b)$ circulation ratios $\tilde{\Gamma}$. (a) $\tilde{\Gamma}=1(-), \tilde{\Gamma}=0.6(---), \tilde{\Gamma}=0.4(-\cdot-)$ and $(b) \tilde{\Gamma}=-1(-), \tilde{\Gamma}=-0.6(---), \tilde{\Gamma}=-0.4(-\cdot-)$.

the steady strain field generated by the strong vortex. The existence of these additional peaks can be attributed mostly to the fact that the self-induction functions of the two vortices are not equal since $F_{h}^{(l)} \neq F_{h}^{(r)}$ in addition to $R o^{(l)} \neq R o^{(r)}$. Indeed, if the two self-induction functions are artificially imposed to be equal, the growth rate curves have generally one single peak but it is located either near the first or the third peak depending whether the largest or the smallest self-induction function is used, respectively.

In the case of unequal-strength counter-rotating vortex pairs (figure 19b), the growth rate remains zero for small wavenumbers and becomes positive only for a finite wavenumber $\hat{k}_{c}$ which is approximately proportional to $1+\tilde{\Gamma}$. The curves then exhibit two maxima. The first one is always small and has a non-zero frequency (not shown). The second peak corresponds to a quasi-antisymmetric eigenmode and derives continuously from the antisymmetric instability existing for $\tilde{\Gamma}=-1$. When the circulation ratio $|\tilde{\Gamma}|$ decreases, the maximum growth rate decreases and the unstable wavenumber range broadens towards high wavenumbers. The latter feature can be attributed again to the difference between the self-induction functions of the two vortices. These results remain qualitatively similar when the Rossby numbers and the Froude numbers are varied provided that $F_{h}^{(l)}<1.83$.

When the two Froude numbers $F_{h}^{(l, r)}$ are larger than 1.83 and $R o^{(l)}=\infty$, the stability of unequal-strength vortex pairs is not very different from the limiting cases $\tilde{\Gamma}= \pm 1$ : co-rotating vortex pairs $(\tilde{\Gamma}>0)$ are always stable while counter-rotating vortex pairs $(\tilde{\Gamma}<0)$ are unstable only to a quasi-symmetric instability.

\section{Instability mechanism}

In the previous section, we have seen that equal-strength counter-rotating vortex pairs are unstable to a quasi-antisymmetric instability in strongly stratified-rotating fluids while the instability is known to be symmetric when the fluid is homogeneous (the Crow instability). Similarly, co-rotating vortex pairs are unstable to a symmetric instability in strongly stratified-rotating fluids whereas it is stable in homogeneous 
fluids. The purpose of this section is to shed light on the origin of these striking differences.

In homogeneous fluids, Saffman (1992) (see also Moore \& Saffman 1975) explains the physical mechanism of the Crow instability by considering the slow bending deformations of a single-vortex filament in a uniform and steady strain. This amounts to neglect the mutual-induction functions in the growth rates (3.3) and (3.4) of an equal-strength counter-rotating vortex pair:

$$
\sigma^{2}=1-\tilde{b}^{4} \omega_{r}^{2}
$$

As argued by Saffman (1992), this shows that the strain is destabilizing whereas the self-induction stabilizes. However, the maximum growth rate occurs when $\omega_{r}=0$, i.e. for two-dimensional perturbations, $\hat{k}=0$, whereas both the Crow instability and the zigzag instability are neutral for $\hat{k}=0$ and most unstable at finite wavenumbers. Furthermore, according to (4.1), the growth rate should be the same for symmetric and antisymmetric modes. Although it points out the destabilizing effect of the strain, this reasoning is therefore too simplified to explain the difference between homogeneous and stratified-rotating fluids and to account for the difference of stability between symmetric and antisymmetric modes.

To go further, the mutual-induction effects should not be neglected. They are indeed comparable to the strain effects for long wavelength since $\Psi \approx \chi \approx 1$ for $\hat{k} \ll 1$. This means that the bending perturbation of one vortex feels as much the perturbation as the strain of the other vortex. It can be noted that this differs from the elliptic instability which can be well described by considering a single vortex in a steady strain field because the perturbation decays much faster than the strain outside the vortex core.

The Crow and zigzag instabilities are most unstable at finite wavenumbers but they are generally of long-wavelength nature, i.e. the growth rate curves start from $\hat{k}=0$ (except for unequal-strength vortex pairs when $-1<\tilde{\Gamma}<0$ ). To understand the instability mechanism, it is therefore interesting to consider the very long wavelength limit $\hat{k} \ll 1$. When $\hat{k}=0$, we have $\Psi=\chi=1$ and $\omega^{(r)}=\omega^{(l)}=0$. There exist two kinds of non-trivial eigenmodes. The first corresponds to displacements such that

$$
\overline{\boldsymbol{\Delta}} \boldsymbol{x}=(0,-\tilde{\Gamma}, 0,1),
$$

with growth rate $\sigma=0$ regardless of the value of $\tilde{\Gamma}$. This eigenmode belongs to the symmetric class for both equal-strength co-rotating and counter-rotating vortex pairs. Physically, it corresponds to a small rotation of the vortex pair with respect to the rotation centre $\left(x_{c}, y_{c}\right)$ and is therefore neutral in the two-dimensional limit due to rotational invariance. When the two vortices of the pair have opposite circulation, $\tilde{\Gamma}=-1$, the rotation centre is located infinitely far from the vortex pair so that the vortex moves in straight line along the $y$ direction. The eigenmode (4.2) then reduces to a small translation in the $y$ direction and is neutral because of translational invariance in the $y$ direction.

The second non-trivial eigenmode in the two-dimensional limit is different according to the value of $\tilde{\Gamma}$ :

$$
\left.\begin{array}{lll}
\bar{\Delta} x=(1, \pm \mathrm{i}, 1, \pm \mathrm{i}) & \text { when } & \tilde{\Gamma} \neq-1, \\
\overline{\boldsymbol{\Delta}} \boldsymbol{x}=(1,0,1,0) & \text { when } & \tilde{\Gamma}=-1,
\end{array}\right\}
$$

with growth rate $\sigma= \pm \mathrm{i}(1+\tilde{\Gamma})$. This eigenmode belongs to the antisymmetric class for both equal-strength co-rotating and counter-rotating vortex pairs. Its growth rate is zero when $\tilde{\Gamma}=-1$ because of translational invariance in the $x$ direction. 


\begin{tabular}{|c|c|c|c|c|}
\hline & \multicolumn{2}{|c|}{ Mode (4.3) } & \multicolumn{2}{|c|}{ Mode (4.2) } \\
\hline & $\tilde{\Gamma}<0$ & $0<\tilde{\Gamma}$ & $\tilde{\Gamma}<0$ & $0<\tilde{\Gamma}$ \\
\hline$R o \rightarrow 0$, stratified fluid $\left(F_{h} \ll \tilde{b}^{2}\right)$ & Unstable $^{*}$ & - & - & Unstable \\
\hline$R o \rightarrow \infty, F_{h}<1.83$ & Unstable* $^{*}$ & - & - & Unstable \\
\hline$R o \rightarrow \infty, 1.83<F_{h} \ll \tilde{b}^{2}$ & - & - & Unstable & - \\
\hline Homogeneous fluid $\left(R o=\infty, F_{h}=\infty\right)$ & - & - & Unstable & - \\
\hline \multicolumn{5}{|c|}{$\begin{array}{l}\text { TABLE 1. Overview of the instability of the modes }(4.3) \text { and }(4.2) \text { (corresponding to the } \\
\text { antisymmetric and symmetric modes, respectively, when }|\tilde{\Gamma}|=1 \text { ) for very long waves as a } \\
\text { function of } \tilde{\Gamma} \text { and the nature of the fluid. The modes are neutral for } \hat{k} \ll 1 \text { unless specified. } \\
\text { The asterisk indicates a mode which is unstable in the very long wave limit only for } \tilde{\Gamma}=-1 \\
\text { but unstable for finite wavenumbers when } \tilde{\Gamma} \neq-1 \text {. }\end{array}$} \\
\hline
\end{tabular}

Two-dimensional displacement perturbations are therefore always neutral. Small three-dimensional effects $\hat{k} \ll 1$ will be able to significantly influence the stability of these perturbations only when $\sigma$ vanishes for $\hat{k}=0$. This is always the case of the mode (4.2) while the mode (4.3) has a zero growth rate only when $\tilde{\Gamma}=-1$. The latter case is particular and will be treated subsequently.

\subsection{Long-wavelength stability of the mode (4.2) when $\tilde{\Gamma} \neq-1$}

When $\tilde{\Gamma} \neq-1$, the growth rate of the mode (4.2) in the very long wavelength limit is at leading order

$$
\sigma^{2}=2 \tilde{\Gamma}\left[2(\Psi-1)+\tilde{b}^{2} \omega_{r}^{(r)}+\tilde{b}^{2} \omega_{r}^{(l)}\right]+O\left(\sigma \tilde{b}^{2} \omega_{i}^{(r, l)},\left(\tilde{b}^{2} \omega^{(r, l)}\right)^{2},(\Psi-1)^{2},(\chi-1)^{2}\right) .
$$

\subsubsection{Stratified non-rotating fluids}

In the particular case of a stratified non-rotating fluid $(R o=\infty)$, we have $\Psi=1$ whatever $\hat{k}$ so that (4.4) directly shows that small three-dimensional effects are destabilizing when the product of $\tilde{\Gamma}$ and the average of the real part of the selfinduction functions of the two vortices is positive. As already mentioned, $\omega_{r}^{(i)}$ is positive when $F_{h}^{(i)}<1.83$ and negative when $F_{h}^{(i)}>1.83$ for Lamb-Oseen vortices for $R o=\infty$. Thus, when the Froude numbers of both vortices are lower than 1.83, corotating vortex pairs, i.e. $\tilde{\Gamma}>0$, will be unstable in the long-wavelength limit whereas counter-rotating vortex pairs, i.e. $\tilde{\Gamma}<0$, will be neutral. The situation is reversed when the Froude numbers of the two vortices are both larger than 1.83 . This fully agrees with the results of $\S 3.2$ where the symmetric mode for $\tilde{\Gamma}=1$ has been found to be unstable for $R o=\infty$ only when $F_{h}<1.83$. For clarity, these stability properties are summarized in table 1 .

\subsubsection{Stratified and rotating fluids}

In the general case of a stratified and rotating fluid, the mutual-induction function $\Psi$ in the long-wavelength limit and the self-induction functions $\omega^{(i)}$ can be written as

$$
\begin{aligned}
\Psi & =1+\frac{\hat{\beta}^{2} b^{2}}{2}\left(\ln \frac{\hat{\beta} b}{2}+\gamma_{e}-\frac{1}{2}\right)+O\left(\hat{\beta}^{3} b^{3}\right), \\
\tilde{b}^{2} \omega^{(i)} & =\frac{\hat{\beta}^{2} b^{2}}{2}\left(-\ln \frac{\hat{\beta} R}{2}-\gamma_{e}+\delta\left(F_{h}^{(i)}, R o^{(i)}\right)\right) .
\end{aligned}
$$


The leading three-dimensional terms in the expressions (4.5) and (4.6) are $O\left(\hat{\beta}^{2} b^{2} \ln \hat{\beta}\right)$ but remarkably these terms cancel out in the growth rate. The first significant terms are therefore $O\left(\hat{\beta}^{2} b^{2}\right)$ :

$$
\sigma^{2}=\tilde{\Gamma} \hat{\beta}^{2} b^{2}\left[2 \ln \tilde{b}-1+\delta_{r}\left(F_{h}^{(l)}, R o^{(l)}\right)+\delta_{r}\left(F_{h}^{(r)}, R o^{(r)}\right)\right]+\cdots,
$$

where $\delta_{r}=\operatorname{Re}(\delta)$. We see that small three-dimensional effects are destabilizing when the product of $\tilde{\Gamma}$ and the term inside the brackets is positive. The unshaded area in figure $18(b)$ for $\tilde{\Gamma}=1$ shows the domain in the parameter space $F_{h}-R o$ where the term inside the brackets is positive. This region exactly corresponds to the unstable domain, demonstrating that the origin of these instabilities can be indeed understood by looking at the long-wavelength limit.

We now consider in detail the regime of rapid background rotation: $R o \leqslant O(1)$. In this case, we have $\delta_{r}=O(1)$ so that the dominant term inside the brackets in (4.7) is $\ln \tilde{b}$ because $\tilde{b} \gg 1$. Therefore, we see that the growth rate will be real and positive in the long-wavelength limit for co-rotating vortex pairs, i.e. $\tilde{\Gamma}>0$, and imaginary for counter-rotating vortex pairs, i.e. $\tilde{\Gamma}<0$, as for the regime $\left(F_{h}<1.83, R o=\infty\right)$ discussed in $\$ 4.1 .1$. These stability properties are also summarized in table 1 . It is interesting to compare this situation to the case of a homogeneous fluid for which (Crow 1970; Widnall, Bliss \& Zalay 1971)

$$
\begin{aligned}
\Psi & =1-\frac{\hat{k}^{2} b^{2}}{2}\left(\ln \frac{\hat{k} b}{2}+\gamma_{e}+\frac{1}{2}\right)+O\left(\hat{k}^{3} b^{3}\right), \\
\tilde{b}^{2} \omega & =\frac{\hat{k}^{2} b^{2}}{2}\left(\ln \frac{\hat{k} R}{2}-\mathscr{D}(0)+\gamma_{e}\right) .
\end{aligned}
$$

This yields

$$
\sigma^{2}=-\hat{k}^{2} b^{2} \tilde{\Gamma}(2 \ln \tilde{b}+2 \mathscr{D}(0)+1)+\cdots
$$

The order $O\left(\hat{k}^{2} b^{2} \ln \hat{k}\right)$ also cancels out in (4.10). Since the term $\ln \tilde{b}$ is again asymptotically dominant, we see that the situation is reversed in homogeneous fluids: counter-rotating vortex pairs, i.e. $\tilde{\Gamma}<0$, are unstable in the long-wavelength limit while co-rotating vortex pairs are stable (i.e. neutral) as reported by Klein, Majda \& Damodaran (1995) and Bristol et al. (2004).

The reason for this reversal of stability between homogeneous and stratified-rotating fluids can be understood by seeking the origin of the term $\ln \tilde{b}$ : this term comes from the fact that the wavenumber in the logarithm is scaled with the vortex radius $R$ in the self-induction function instead of the separation distance $b$ in the mutual-induction functions. Since $\hat{k} R \ll \hat{k} b \ll 1$, the order $O\left(\hat{k}^{2}\right)$ of the self-induction functions (4.6) and (4.9) is much larger than the one of the mutual-induction functions (4.5) and (4.8), and therefore controls the sign of the growth rate in the long-wavelength limit. Hence, the exchange of stability between the two types of fluid can be traced back to the reversal of the sign of the self-induction function: positive for stratified and rotating fluids for $R o \leqslant O(1)$ and negative for homogeneous fluids.

\subsection{Equal-strength counter-rotating vortex pairs}

In the particular case $\tilde{\Gamma}=-1$, both the mode (4.2), which is symmetric, and the mode (4.3), which is antisymmetric, have a zero growth rate when $\hat{k}=0$. As a consequence, they both can become unstable for small vertical wavenumber. For simplicity, we restrict the discussion to the cases of homogeneous fluids and stratified-rotating fluids 
for $R o \rightarrow 0$ or $R o \rightarrow \infty$. In these limits, the self-induction functions of the two vortices are equal, $\omega^{(l)}=\omega^{(r)}=\omega$ so that antisymmetric and symmetric modes are decoupled even when $\hat{k} \neq 0$. Their growth rates in the very long wavelength limit are

$$
\begin{aligned}
& \sigma_{s}^{2}=2(1-\Psi)-2 \tilde{b}^{2} \omega_{r}+\cdots, \\
& \sigma_{a}^{2}=2(1-\chi)+2 \tilde{b}^{2} \omega_{r}+\cdots .
\end{aligned}
$$

The expression (4.11) of the growth rate of the symmetric mode differs from (4.4) only by a factor 2 . The results derived in $\S 4.1$ for $\tilde{\Gamma} \neq-1$ therefore also apply for $\tilde{\Gamma}=-1$ (see table 1$)$.

Regarding the antisymmetric mode, the mutual-induction function $\chi$ in a stratified and rotating fluid is

$$
\chi=1-\frac{\hat{\beta}^{2} b^{2}}{2}\left(\ln \frac{\hat{\beta} b}{2}+\gamma_{e}+\frac{1}{2}\right)+O\left(\hat{\beta}^{3} b^{3}\right),
$$

so that (4.12) becomes

$$
\sigma_{a}^{2}=\hat{\beta}^{2} b^{2}\left(\ln \tilde{b}+\delta_{r}\left(F_{h}, R o\right)+\frac{1}{2}\right)+\cdots .
$$

In homogenous fluids, we have

$$
\chi=1+\frac{\hat{k}^{2} b^{2}}{2}\left(\ln \frac{\hat{k} b}{2}+\gamma_{e}-\frac{1}{2}\right)+O\left(\hat{k}^{3} b^{3}\right)
$$

leading to

$$
\sigma_{a}^{2}=-\hat{k}^{2} b^{2}\left(\ln \tilde{b}+\mathscr{D}(0)-\frac{1}{2}\right)+\cdots .
$$

As summarized in table 1 , the antisymmetric mode (mode (4.3) for $\tilde{\Gamma}=-1$ ) is therefore neutral in homogeneous fluids in the long-wavelength limit, whereas it is unstable in stratified and rotating fluids for $R o \rightarrow 0$ whatever the Froude number (provided that the theory is valid, i.e. $F_{h} \ll \tilde{b}^{2}$ ). When $R o \rightarrow \infty$, the antisymmetric mode is unstable for $F_{h}<1.83$ and neutral for $F_{h}>1.83$. The stability properties of the antisymmetric mode are therefore opposite to those of the symmetric mode. Although this analysis is restricted to very long waves, it is in full agreement with the results of $\S 3.1$ for $\tilde{\Gamma}=-1$.

When $-1<\tilde{\Gamma}<0$, the mode (4.3) actually continues to be unstable but only for finite wavenumbers $\hat{k}>\hat{k}_{c}$, as found in $\S 3.4$. Nevertheless, the present long-wavelength analysis can be extended to treat this case when the circulation ratio is close to -1 . It shows that the cutoff wavenumber $\hat{k}_{c}$ scales as $\hat{k}_{c} F_{h}^{(l)} b \propto(1+\tilde{\Gamma})$.

\subsection{Physical interpretation}

\subsubsection{Symmetric mode}

In fact, it is easy to understand physically why the sign of the self-induction controls the stability of vortex pairs. This is illustrated in figure 20 in the case of the symmetric mode for an equal-strength counter-rotating vortex pair $\tilde{\Gamma}=-1$. We have slightly perturbed the vortex pair by sinusoidally bending the two vortices in the direction of propagation (figure 20a). Such perturbation corresponds to the symmetric mode in the limit of an infinite wavelength (see (4.2)). The three effects acting on the perturbed vortices are shown in a horizontal cross-section for homogeneous fluids (figure $20 b$ ) and stratified fluids (figure 20c). (For simplicity, we assume that $F_{h}<1 / \Omega_{\max }$ so that 
(b)
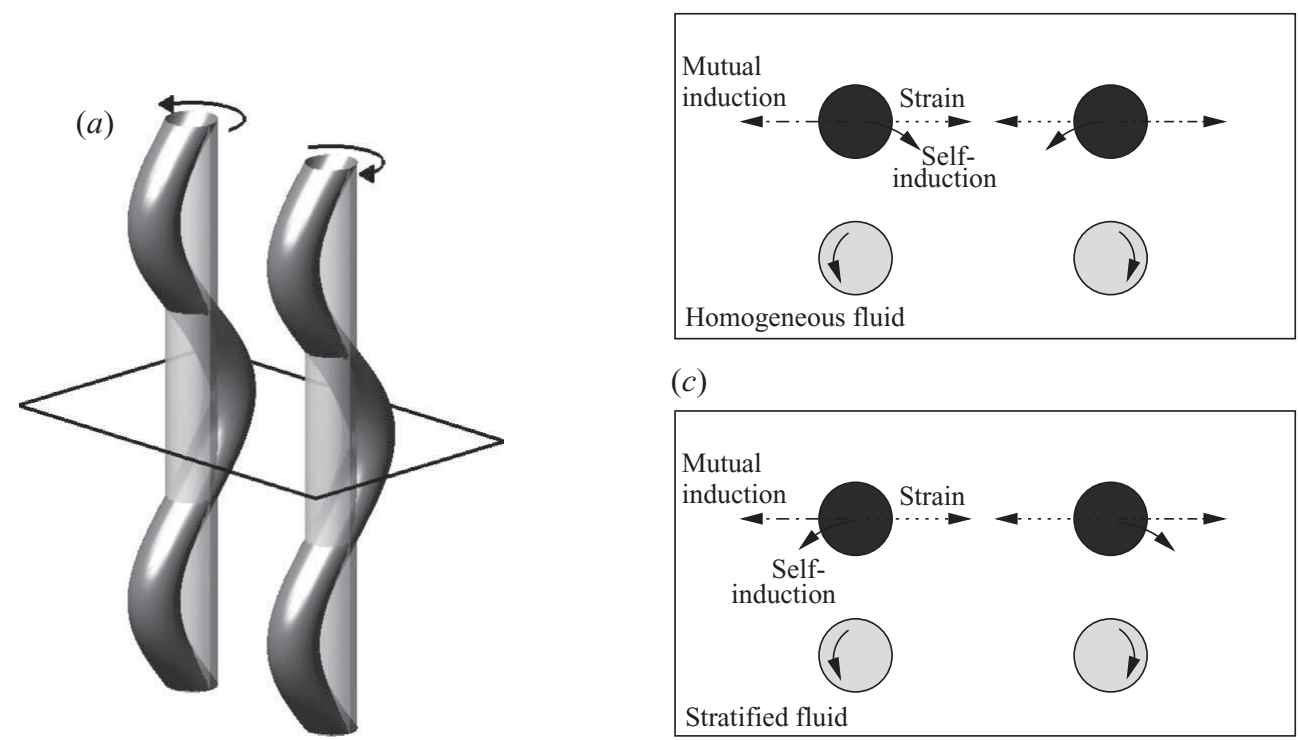

(c)

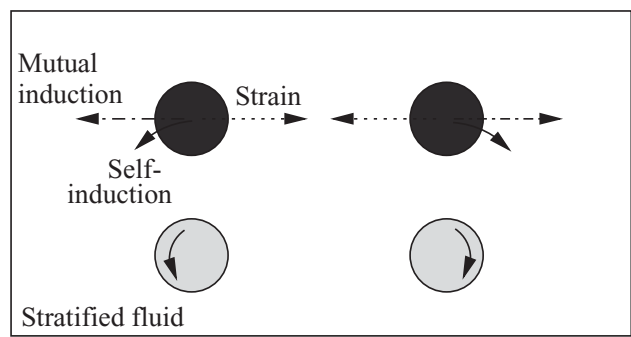

FiguRE 20. Physical interpretation of the stability of the symmetric mode for an equal-strength counter-rotating vortex pair. (a) The two vortices (shown by heavy grey) have been bent sinusoidally in the direction of propagation. The unperturbed vortices are represented in light grey. The effects acting on the perturbed vortices in the horizontal cross-section shown in $(a)$ are displayed in $(b)$ for homogeneous fluids and in $(c)$ for stratified fluids. The dashed-dotted arrows show the mutual-induction effect, the dotted arrows represent the effect of the strain and finally the solid curved arrows show the effect of the self-induction.

there is no critical layer and a non-rotating fluid: $R o=\infty$.) In this cross-section, the vortex pair has been displaced forward. In both types of fluids, the effect of the strain is to bring closer the two vortices whereas the mutual induction tends the two vortices to move apart. When the wavelength is infinite, these two effects exactly counterbalance each other so that the net effect is zero since the self-induction is also zero in this case. The applied perturbations are therefore neutral, reflecting the translational invariance in the $y$ direction. Now, for long wavelength, the mutual induction differs slightly from the strain and the self-induction is no longer zero. However, as seen previously, the self-induction is dominant and therefore governs the net effect.

Since the self-induction is negative in homogeneous fluids, it tends to displace each vortex perpendicularly to the displacement perturbation in the opposite direction of rotation of the vortex. Figure $20(b)$ shows that its effect is therefore to bring closer the two vortices in the cross section plane. As the velocity of propagation of the vortex pair is inversely proportional to the separation distance, the vortex pair will, in turn, propagate slightly faster, leading to an amplification of the initial perturbation. Conversely, in the regions where the vortices have been displaced backwards, the vortex pair will travel slower, amplifying the initial disturbance also. This is the physical mechanism at the origin of the Crow instability of counter-rotating vortex pairs in homogeneous fluids.

In contrast, since the self-induction is positive in stratified fluids (figure $20 c$ ), the effects described above are reversed: the two vortices move apart (respectively closer) in the regions where they have been displaced forwards (respectively backwards). Thus, 

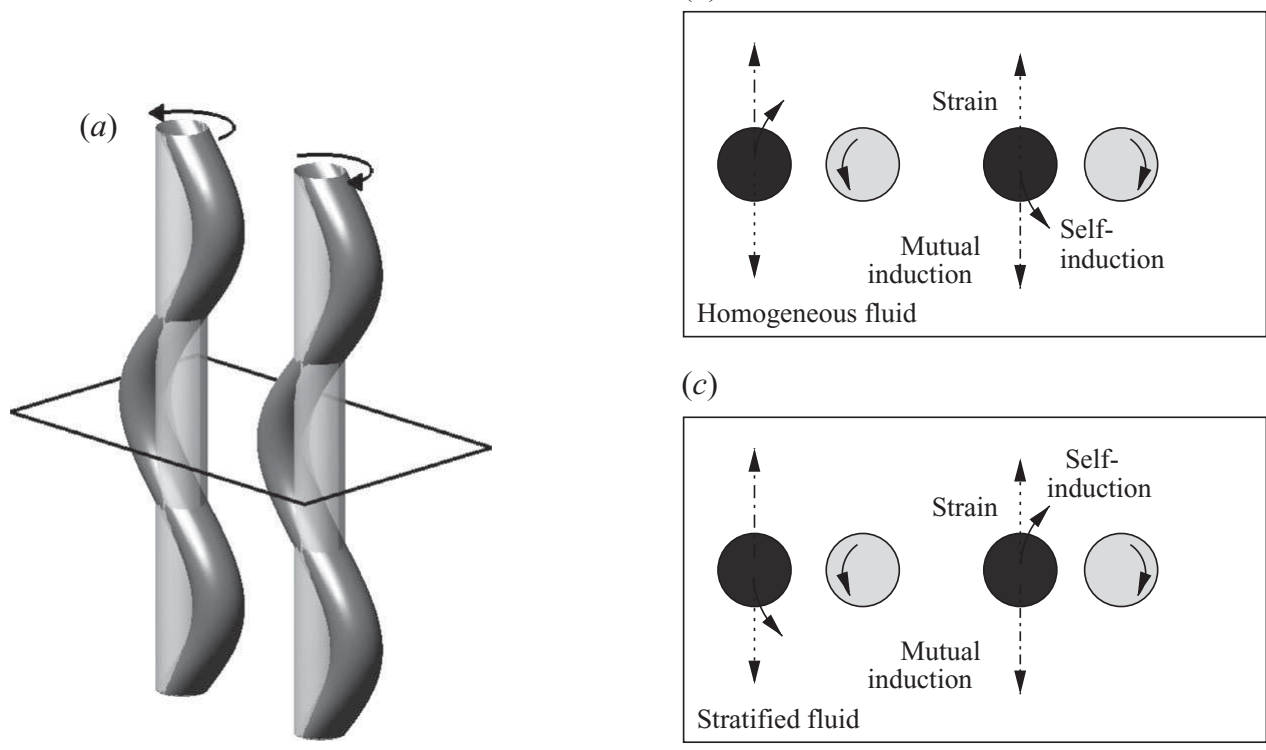

(c)

FIGURE 21. Similar to figure 20 but for the antisymmetric mode.

the propagation velocity of the vortex pair varies such that the initial perturbation is reduced. This explains why the symmetric mode is stable in stratified fluids.

The stability of the mode (4.2) when $\tilde{\Gamma} \neq-1$ can be understood similarly.

\subsubsection{Antisymmetric mode}

A similar reasoning can explain the stability of the antisymmetric mode for $\tilde{\Gamma}=-1$. As sketched in figure 21( $a$ ), the vortex pair is now perturbed by sinusoidally bending the two vortices in the direction perpendicular to the direction of propagation. When the wavelength is infinite, this perturbation corresponds to the antisymmetric mode (see (4.3)) which is neutral due to the translational invariance in the $x$ direction. For long wavelength, the strain and mutual induction almost counter-balance each other like for the symmetric mode and the dominant effect is the self-induction. In stratified fluids (figure 21c), we see that the self-induction tends to move backwards the left vortex and forward the right vortex. The vortex pair will thus rotate anticlockwise and have a small component of velocity leftwards in a region where it has been moved initially leftwards. The initial perturbation will thus increase. Conversely, in the regions where the vortex pair has been displaced rightwards, the vortex pair will start to move rightwards. This is the physical mechanism at the origin of the zigzag instability. This mechanism is consistent with the explanations given by Billant \& Chomaz (2000b) for the specific case of the Lamb-Chaplygin vortex pair although they have not separately considered the effects on each vortex. In contrast, the antisymmetric mode is stable in homogeneous fluids (figure 21b) since the direction of the self-induction is reversed.

\section{Conclusions}

In this paper, we have theoretically and numerically investigated the stability of equal-strength co-rotating and counter-rotating columnar vertical Lamb-Oseen vortex pairs in a stratified and rotating fluid. The theory, which has been developed in Part 1, 
describes the interactions between the strain and the long-wavelength bending deformations of each vortex column. It is valid for well-separated vortices and when the strain is much smaller than the Brunt-Väisälä frequency.

The theoretical predictions are in excellent agreement with the results of direct numerical stability analyses except for equal-strength co-rotating vortex pairs with the separation ratio $\tilde{b}=6.7$ around the Rossby number $R o \approx-2$. However, there is a significant improvement of the theory if a larger separation ratio $\tilde{b}$ is considered or if higher-order three-dimensional effects are taken into account by means of an improved asymptotic approach.

We have therefore been able to draw a complete picture of the stability properties of vortex pairs in stratified and rotating fluids with respect to long-wavelength bending disturbances. Equal-strength co-rotating vortex pairs are unstable to a symmetric instability when the Froude number $F_{h}$ is lower than a critical Froude number, which mainly depends on the Rossby number. Equal-strength counter-rotating vortex pairs are always unstable: when $F_{h}$ is lower than a critical Froude number depending mostly on the Rossby number, the instability is quasi-antisymmetric. When $F_{h}$ is above this threshold, the instability becomes quasi-symmetric. There is no exact decoupling between symmetric and antisymmetric modes for finite Rossby number because the self-induction functions of the cyclonic and anticyclonic vortices are different. Accordingly, the cyclonic vortex tends to be less bent than the anticyclonic vortex except when $R o \rightarrow 0$ or $R o \rightarrow \infty$.

These bending instabilities are most unstable for $F_{h}<1$ : the maximum growth rate is approximately equal to the strain $\Gamma /\left(2 \pi b^{2}\right)$ for counter-rotating vortex pairs and equal to twice the strain for co-rotating vortex pairs independently of $F_{h}$ and $R o$. The corresponding most amplified wavelength scales like $F_{h} b / f(R o)$ where the function $f(R o)$ is such that $f(R o) \rightarrow$ const. for $R o \rightarrow \infty$ and $f(R o) \rightarrow R o$ for $R o \rightarrow 0$. The damping by viscous and diffusive effects in this regime varies strongly with the Rossby and Froude numbers: for given Reynolds and Schmidt numbers, the maximum growth rate is reduced as the Rossby number $|R o|$ increases or as the Froude number decreases.

When $F_{h}>1$, the maximum growth rate is much lower because the slow bending modes are damped by a critical layer at the radius where the angular velocity of the vortex is equal to the Brunt-Väisälä frequency. The critical-layer solution derived in Part 1 is in striking agreement with the numerical results. In spite of this damping, these bending instabilities remain most dangerous in the range of parameters investigated in the direct numerical stability analyses. They are therefore of particular importance to understand the dynamics of vortices in stratified-rotating flows. Nevertheless, it should be stressed that other types of instabilities such as the elliptic instability or the centrifugal instability may exist in other regions of the parameter space, especially for high Froude numbers or large vertical wavenumbers.

It is also worth mentioning that the critical layer always acts to damp the exponential growth of the bending instabilities since we are in a linear-stability framework. However, Boulanger, Meunier \& Le Dizès (2008) have shown for the case of a stratified tilted vortex that the strong vertical motions generated inside the critical layer can be unstable to shear instabilities for large Reynolds number and sufficient vortex inclination. This suggests that once the zigzag instability has reached a sufficient amplitude, secondary shear instabilities could develop in the critical layer in the regions where the vortices are the most bent. A study of the nonlinear evolution of the zigzag instability in the regime $F_{h}>1 / \Omega_{\max }$ and for large Reynolds number would be necessary to investigate this hypothesis. 
The self-induction function and particularly its sign has been shown to account for the distinct stability properties of vortex pairs in homogeneous and stratifiedrotating fluids. The self-induction function is indeed positive in stratified and rotating fluids when $F_{h}<1$ while it is negative in homogeneous fluids. Such sign reversal explains why equal-strength counter-rotating vortex pairs are subjected to a quasiantisymmetric instability in stratified-rotating fluids (zigzag instability or tall-column instability) and to a symmetric instability in homogeneous fluids (the Crow instability). It explains similarly why equal-strength co-rotating vortex pairs are unstable to a symmetric zigzag instability in stratified and rotating fluids whereas they are stable in homogeneous fluids.

We would like to thank D. Guy for technical assistance and the referees for their helpful comments and suggestions.

\section{Appendix. Effect of the periodic boundary conditions in the numerical stability analyses}

The direct numerical stability analyses have been carried out with a pseudospectral code and thus periodic boundary conditions (see $\S 2.2$ ). If the computational box is of size $L_{x} \times L_{y}$, this implies that the vortex pair actually repeats itself along the $x$ direction with a period $L_{x}$ and in the $y$ direction with a period $L_{y}$. The effects of the image vortices are minimized by taking a large computational box. However, the theory for an arbitrary vortex array (Part 1) offers the possibility of simply testing the influence of the periodic boundary conditions. To do so, we consider an infinite vortex array like in the computational box: there is a vortex of circulation $\Gamma^{(l)}$ at every position $(x, y)=\left(n L_{x}, p L_{y}\right)$ and a vortex of circulation $\Gamma^{(r)}$ at every position $(x, y)=\left(n L_{x}+b, p L_{y}\right)$, where $n$ and $p$ range over all integral values. The displacement perturbations of all the vortices labelled $(l)$ are imposed to be equal and similarly for the vortices labelled $(r)$.

Under these hypotheses, the equations for the displacement perturbations $\left(\Delta x^{(l)}, \Delta y^{(l)}\right)$ remain the same as $(2.5)-(2.6)$, except that we must add the following terms: $-P \Delta y^{(l)}+Q \Delta y^{(r)}$ and $-\tilde{P} \Delta x^{(l)}+\tilde{Q} \Delta x^{(r)}$ to the right-hand sides of $(2.5)$ and (2.6), respectively, where $P$ and $Q$ are given by

$$
\begin{gathered}
P=\frac{\Gamma^{(r)}}{2 \pi} \sum_{n \neq 0} \frac{1}{L_{r 0 n}^{2}}+\frac{\Gamma^{(l)}}{2 \pi} \sum_{n \neq 0} \frac{1-\Psi_{l 0 n}}{L_{l 0 n}^{2}}+\frac{\Gamma^{(r)}}{2 \pi} \sum_{p \neq 0} \sum_{n} \frac{\left(n L_{x}+b\right)^{2}-p^{2} L_{y}^{2}}{L_{r p n}^{4}} \\
+\frac{\Gamma^{(l)}}{2 \pi} \sum_{p \neq 0} \sum_{n} \frac{n^{2} L_{x}^{2}\left(1-\Psi_{l p n}\right)-p^{2} L_{y}^{2}\left(1-\chi_{l p n}\right)}{L_{l p n}^{4}}, \\
Q=\frac{\Gamma^{(r)}}{2 \pi} \sum_{n \neq 0} \frac{\Psi_{r 0 n}}{L_{r 0 n}^{2}}+\frac{\Gamma^{(r)}}{2 \pi} \sum_{p \neq 0} \sum_{n} \frac{\left(n L_{x}+b\right)^{2} \Psi_{r p n}-p^{2} L_{y}^{2} \chi_{r p n}}{L_{r p n}^{4}}
\end{gathered}
$$

where $L_{r p n}=\left(p^{2} L_{y}^{2}+\left(n L_{x}+b\right)^{2}\right)^{1 / 2}, L_{l p n}=\left(p^{2} L_{y}^{2}+n^{2} L_{x}^{2}\right)^{1 / 2}$ and $\Psi_{r p n}=\Psi\left(\hat{\beta} L_{r p n}\right)$, $\chi_{r p n}=\chi\left(\hat{\beta} L_{r p n}\right)$ and $\Psi_{l p n}=\Psi\left(\hat{\beta} L_{l p n}\right), \chi_{l p n}=\chi\left(\hat{\beta} L_{l p n}\right)$. The coefficients $\tilde{P}$ and $\tilde{Q}$ are found by interchanging the symbols $\Psi$ and $\chi$ in $P$ and $Q$. The terms that must be added to the right-hand sides of the equations for the vortex $(r)$ are found by interchanging the superscripts $(r)$ and $(l)$ in the expressions given above.

The theoretical growth rate taking into account the periodic boundary conditions are plotted as dashed lines in figure $22(a)$. Otherwise, this figure is the same as figure 1 

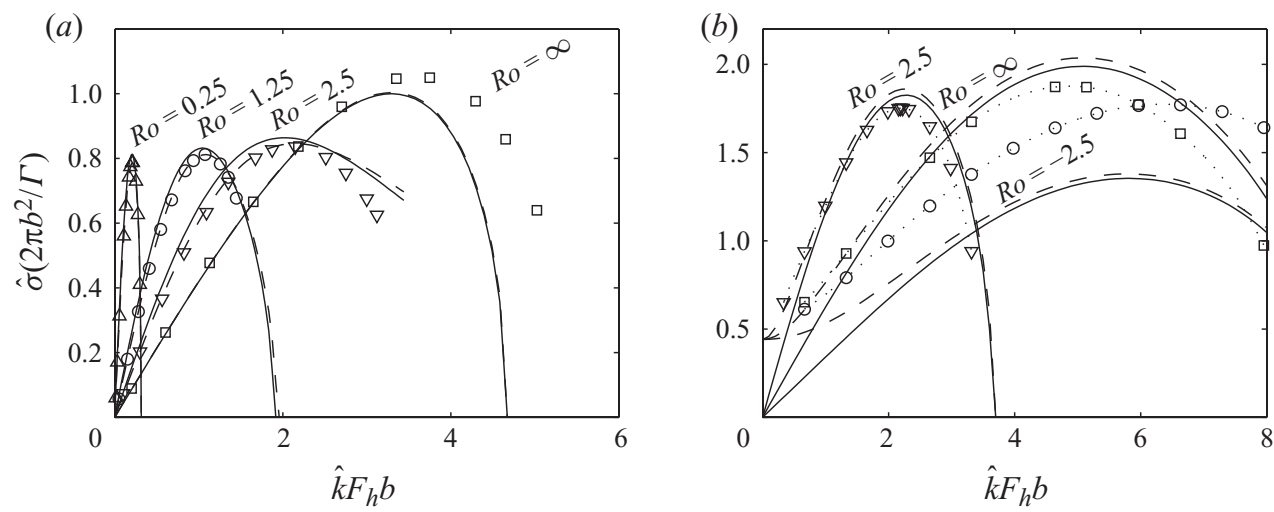

FIGURE 22. (a) Similar to figure 1 except that the theoretical growth rate taking into account the periodic boundary conditions has also been plotted with dashed lines for the sizes $L_{x}=38 R$ and $L_{y}=30 R$ used in the numerical stability analysis. (b) Similar to figure 14 except that the theoretical growth rate taking into account the periodic boundary conditions has also been plotted with dashed lines for the sizes $L_{x}=L_{y}=30 R$ used in the numerical stability analysis of Otheguy et al. (2006a). Only the curves for the Rossby numbers $R o=\infty(\square), R o=-2.5(\bigcirc)$ and $R o=2.5(\nabla)$ have been plotted.

for an equal-strength counter-rotating vortex pair with $\tilde{b}=8$ and $F_{h}=0.1$. We see that the differences with the theoretical growth rate for an isolated vortex pair (solid lines) are very weak. The solid and dashed curves are almost superposed for $R o=\infty$ and $R o=0.25$ whereas some differences are visible for $R o=2.5$ and $R o=1.25$. Quite remarkably, the dashed curves are in closer agreement with the numerical results than the solid curves for low and moderate vertical wavenumbers.

Similarly, figure $22(b)$ is similar to figure 14 for the case of an equal-strength corotating vortex pair for $R o=\infty, R o=-2.5$ and $R o=2.5$ except that the theoretical growth rate taking into account the periodic boundary conditions used in the numerical stability analysis of Otheguy et al. (2006a) has also been plotted with dashed lines. We see that the theoretical growth rate with periodic boundary conditions is no longer zero for $\hat{k}=0$ but approximately 0.5 and agrees better with the numerical results for small $\hat{k}$. It is thus very different from the case of an isolated vortex pair and explains why the growth rate obtained numerically does not seem to vanish for $\hat{k}=0$ in figures 13 and 14 . However, for larger wavenumbers and in particular for the most amplified wavenumber, the effect of the periodic boundary conditions becomes small. Overall, this demonstrates that the effects of the periodic boundary conditions used in the numerical stability analysis are generally small when the computational domain is large but not always.

\section{REFERENCES}

Abramowitz, M. \& Stegun, I. A. 1965 Handbook of Mathematical Functions. Dover.

Billant, P. 2010 Zigzag instability of vortex pairs in stratified and rotating fluid. Part 1. General stability equations. J. Fluid Mech. doi:10.1017/S0022112010002818.

Billant, P. \& Chomaz, J.-M. 2000a Experimental evidence for a new instability of a vertical columnar vortex pair in a strongly stratified fluid. J. Fluid Mech. 418, 167-188.

Billant, P. \& Chomaz, J.-M. $2000 b$ Theoretical analysis of the zigzag instability of a vertical columnar vortex pair in a strongly stratified fluid. J. Fluid Mech. 419, 29-63. 
Billant, P. \& Chomaz, J.-M. 2000c Three-dimensional stability of a vertical columnar vortex pair in a stratified fluid. J. Fluid Mech. 419, 65-91.

Billant, P. \& Chomaz, J.-M. 2001 Self-similarity of strongly stratified inviscid flows. Phys. Fluids 13, $1645-1651$.

Billant, P., Dritschel, D. G. \& Chomaz, J.-M. 2006 Bending and twisting instabilities of columnar elliptical vortices in a rotating strongly stratified fluid. J. Fluid Mech. 561, 73-102.

Boulanger, N., Meunier, P. \& Le Dizès, S. 2008 Tilt-induced instability of a stratified vortex. J. Fluid Mech. 596, 1-20.

Brethouwer, G., Billant, P., Lindborg, E. \& Chomaz, J.-M. 2007 Scaling analysis and simulation of strongly stratified turbulent flows. J. Fluid Mech. 585, 343-368.

Bristol, R. L., Ortega, J. M., Marcus, P. S. \& Savas, O. 2004 On cooperative instabilities of parallel vortex pairs. J. Fluid Mech. 517, 331-358.

Crow, S. C. 1970 Stability theory for a pair of trailing vortices. AIAA J. 8, 2172-2179.

Deloncle, A., Billant, P. \& Chomaz, J.-M. 2008 Nonlinear evolution of the zigzag instability in stratified fluids: a shortcut on the route to dissipation. J. Fluid Mech. 599, 229-239.

Dritschel, D. G. \& DE LA TORRE Jú́Rez, M. 1996 The instability and breakdown of tall columnar vortices in a quasi-geostrophic fluid. J. Fluid Mech. 328, 129-160.

Fincham, A. M., Maxworthy, T. \& Spedding, G. R. 1996 Energy dissipation and vortex structure in freely decaying, stratified grid turbulence. Dyn. Atmos. Oceans 23, 155-169.

Godeferd, F. S. \& Staquet, C. 2003 Statistical modelling and direct numerical simulations of decaying stably stratified turbulence. Part 2. Large-scale and small-scale anisotropy. J. Fluid Mech. 486, 115-159.

Hebert, D. A. \& DeBruynKops, S. M. 2006 Relationship between vertical shear rate and kinetic energy dissipation rate in stably stratified flows. Geophys. Res. Lett. 33, L06602.

Herring, J. R. \& MÉtais, O. 1989 Numerical experiments in forced stably stratified turbulence. J. Fluid Mech. 202, 97-115.

JimeneZ, J. 1975 Stability of a pair of co-rotating vortices. Phys. Fluids 18, 1580-1582.

Klein, R., Majda, A. J. \& Damodaran, K. 1995 Simplified equations for the interaction of nearly parallel vortex filaments. J. Fluid Mech. 288, 201-248.

Leblanc, S. 2003 Internal wave resonances in strain flows. J. Fluid Mech. 477, 259-283.

Leweke, T. \& Williamson, C. H. K. 1998 Cooperative elliptic instability of a vortex pair. J. Fluid Mech. 360, 85-119.

LiLLY, D. K. 1983 Stratified turbulence and the mesoscale variability of the atmosphere. J. Atmos. Sci. 40, 749-761.

Lilly, D. K., Bassett, G., Droegemeier, K. \& Bartello, P. 1998 Stratified turbulence in the atmospheric mesoscales. Theor. Comput. Fluid Dyn. 11 (3-4), 139-153.

LindBorg, E. 2006 The energy cascade in a strongly stratified fluid. J. Fluid Mech. 550, 207-242.

Lindborg, E. \& Brethouwer, G. 2007 Stratified turbulence forced in rotational and divergent modes. J. Fluid Mech. 586, 83-108.

Meunier, P. \& Leweke, T. 2005 Elliptic instability of a co-rotating vortex pair. J. Fluid Mech. 533, $125-159$.

Moore, D. W. \& Saffman, P. G. 1975 The instability of a straight vortex filament in a strain field. Proc. R. Soc. Lond. 346, 413-425.

Otheguy, P., Billant, P. \& Chomaz, J. M. $2006 a$ The effect of planetary rotation on the zigzag instability of co-rotating vortices in a stratified fluid. J. Fluid Mech. 553, 273-281.

Otheguy, P., Billant, P. \& Chomaz, J. M. 2007 Theoretical analysis of the zigzag instability of a vertical co-rotating vortex pair in a strongly stratified fluid. J. Fluid Mech. 584, 103-123.

Otheguy, P., Chomaz, J. M. \& Billant, P. $2006 b$ Elliptic and zigzag instabilities on co-rotating vertical vortices in a stratified fluid. J. Fluid Mech. 553, 253-272.

Park, Y.-G., Whitehead, J. A. \& GNANAdEskian, A. 1994 Turbulent mixing in stratified fluids: layer formation and energetics. J. Fluid Mech. 279, 279-311.

Praud, O., Fincham, A. M. \& Sommeria, J. 2005 Decaying grid turbulence in a strongly stratified fluid. J. Fluid Mech. 522, 1-33.

Riley, J. J. \& DeBRuYnKops, S. M. 2003 Dynamics of turbulence strongly influenced by buoyancy. Phys. Fluids 15 (7), 2047-2059. 
Riley, J. J. \& Lelong, M.-P. 2000 Fluid motions in the presence of strong stable stratification. Annu. Rev. Fluid Mech. 32, 617-657.

RiLeY, J. J. \& LiNDBORG, E. 2008 Stratified turbulence: a possible interpretation of some geophysical turbulence measurements. J. Atmos. Sci. 65 (7), 2416-2424.

Riley, J. J., Metcalfe, W. \& Weissman, M. A. 1981 Direct numerical simulations of homogeneous turbulence in density-stratified fluids. In Proceedings of the AIP Conference on Nonlinear Properties of Internal Waves (ed. B. J. Bruce), pp. 79-112. American Institute of Physics.

Saffman, P. G. 1992 Vortex Dynamics. Cambridge University Press.

Waite, M. L. \& Bartello, P. 2004 Stratified turbulence dominated by vortical motion. J. Fluid Mech. 517, 281-308.

Waite, M. L. \& Smolarkiewicz, P. K. 2008 Instability and breakdown of a vertical vortex pair in a strongly stratified fluid. J. Fluid Mech. 606, 239-273.

Widnall, S. E., Bliss, D. \& Zalay, A. 1971 Theoretical and experimental study of the stability of a vortex pair. In Aircraft Wake Turbulence (ed. A. Goldburg, J. H. Olsen \& M. Rogers), pp. 305-329. Plenum. 